\title{
Dancing Chief in the Brain or Consciousness as an Entanglement
}

\author{
Yukio-Pegio Gunji ${ }^{1} \cdot$ Kyoko Nakamura $^{2}$
}

Published online: 18 February 2019

(c) The Author(s) 2019

\begin{abstract}
Free will in intentional consciousness is exposed to skeptics since it was found that subconscious neural activities, what is called readiness potential, precedes the intention to an action. The question of whether free will is an authentic illusion has been argued not only in psychology but physics and philosophy. Most of scientists, however, think that the intentional consciousness who believes to have his/her own free will, is determined by readiness potential in advance, and that free will cannot coexist with determinism. We here point out that knowing to be determined in advance cannot be verified till local event at a local site can be known from a different local site without disturbing the event. That is the assumption of locality. We here show that determinism, free will and locality are three essential elements in consciousness, and show that they constitute trilemma. The fact that one of three elements must be abandoned can lead to three types of consciousness. Absence of free will can constitute type I consciousness which is consistent with symptoms in autism spectrum disorder such as weakened theory of mind. Absence of determinism can constitute type II consciousness which is consistent with symptoms in schizophrenia such as thought insertion, self-other integration. Absence of locality can constitute type III consciousness which is consistent with typical people. We can find the entanglement of intentional consciousness with unconsciousness including readiness potential only in type III consciousness. Finally, we show that sense of agency and free will cannot be established until consciousness as an entanglement is implemented.
\end{abstract}

Keywords Free will $\cdot$ Entanglement $\cdot$ ASD $\cdot$ Schizophrenia $\cdot$ Sense of agency

Yukio-Pegio Gunji

pegioyukio@gmail.com

1 Department of Intermedia, Art and Science, School of Fundamental Science and Technology, Waseda University, Tokyo, Japan

2 Research Institute for Language and Cultures of Asia and Africa, Tokyo University of Foreign Studies, Tokyo, Japan 


\section{Introduction}

Since Libet et al. (1983) showed that readiness potential preparing motor action precedes the action of intentional consciousness, the phenomenon in which the future could affect the past has been investigated through various experiments. When multiple stimuli, for example, auditory and visual, are integrated in the human brain, signals transmitted quickly wait for those transmitted slowly. This could result in contraction and expansion of temporal duration (Eagleman and Holcombe 2002). Since different timelines in a universe (e.g., neural networks) cannot be compared, timelines could comprise partial order rather than linear order (Moore 1988; Sorkin 1991; Markopoulou 2000). Therefore, contraction and/ or expansion of some duration can bring about time reversal or create a scenario wherein the future may affect the past (Cunningham et al. 2001; Tse et al. 2004; Stetson et al. 2006). These phenomena are known as backward perceptional phenomena, such as apparent motion (Kolers and von Grunau 1975; Kolers and von Grünau 1976), cutaneous rabbit (Geldard and Sherrick 1972), flash lag effect (Eagleman 2004; Eagleman et al. 2005), and backward filling-in (Kamitani and Shimojo 1999). Intentional consciousness is regarded as an a-posteriori interpretation or as postdiction (Libet 2004; Shimojo 2014).

Backward perception can give rise to serious problems regarding free will. Intention to move one's finger appears in intentional consciousness approximately $0.350 .5 \mathrm{~s}$ after neurons employed for readiness potential to move that finger are fired. In other words, voluntary action is implicitly and passively created by neurons employed by unconsciousness. In this sense, the significance of voluntary action and/or free will must be shaken. As bodily consciousness is considered to be obtained through the sense of self-agency (Gallagher 2007; Haggard et al. 2002a; Tsakiris et al. 2006; Paillard 1999; Botvinick and Cohen 1998; Farrer and Frith 2002), matching intentional plan to move and motor command plays a central role in generating sense of agency (SoA) and sense of ownership (SoO). The underlying mechanism is expressed as a comparator model in which matching among planned moves, motor command, and the body's actual moves are estimated by feedback and feedforward information processing (Frith et al. 2000; Blakemore et al. 2000, 2002; Wolpert and Flanagan 2001; Gallagher 2000). Matching preceding motor command and intentional plan does not differ from matching following motor command and intentional plan. Therefore, the comparator model is not influenced by postdiction so far as it remains in the concept of matching.

Gallagher (2000) confessed that the comparator model assumes the immune principle of self, in which a self as a norm can compare, in advance, some state with another state. In this sense, the comparator model cannot be influenced by the concept of postdiction. However, we believe that self is generated through the acquisition of SoA and SoO, which are enhanced through self. In other words, self, who can compare some state with another state, could be generated, degenerated, and dynamically sustained through both the distinction and integration of intentional consciousness and unconsciousness including readiness potential. In this approach, bodily consciousness itself is immediately relevant for postdiction. The question posed is as follows: how can one implement the relation between intentional consciousness and unconsciousness featuring circular causation rather than linear causation? (Freeman 2000; Freeman and Vitiello 2006). The relation between intentional consciousness and unconsciousness is the context in which free will and/or determinism can be established and sustained.

One of the most hopeful ways to implement such a context starts from the trilemma of free will, determinism, and locality. This trilemma appears in the free will theorem 
proposed by Conway and Kochen (2006) and Conway and Kochen (2009). In this theorem, locality is given up because quantum physics is accepted. The theorem claims that free will in particles exists if one accepts the free will of an observer who measures the state of particles. Both free will and determinism can hold if one gives up locality. Independent of quantum mechanics (Gunji et al. 2017), analytical philosopher Dummett (1978) also proposed this trilemma. He considered the free will of a chief who attempts to change the pasta situation that can lead to the trilemma of free will, determinism, and locality. Here, we show that the chief's situation can be found in the brain in a postdictive manner and that considering the trilemma can resolve the issue of free will and/or bodily consciousness in postdiction.

Recently, cognitive experiments on bodily consciousness have been conducted and adapted to people with autism spectrum disorder (ASD) (David et al. 2008; Grynszpan et al. 2012; Gowen and Hamilton 2013; Sperduti et al. 2014) and those with schizophrenia (Corbera et al. 2013; Smith et al. 2014; van der Weiden et al. 2013; Renes et al. 2015). After the intentional binding technique was invented, explicit and objective data have been obtained for estimating SoA (Haggard et al. 2002a, b; Haggard and Clark 2003; Lau et al. 2004). Under and/or overestimation of bodily consciousness in patients with ASD and schizophrenia are observed, and results can be immediately relevant for self-other relationships (Kaiser and Shiffrar 2009; Happé and Frith 1996; Baron-Cohen 1995; Baron-Cohen et al. 1985). Also reported is the antisymmetric structure of symptoms of schizophrenia and ASD. In an individual with schizophrenia, separation of intentional consciousness and other neural activities could entail finding others within the self. Fusion of the self and actual others outside the self could entail self-other integration (Synofzik et al. 2013; Abu-Akel and Shamay-Tsoory 2013). Antisymmetric structure is found as internal separation versus external fusion. In contrast, ASD can reveal antisymmetric structure as internal fusion versus external separation. In ASD, internal fusion can bring about a self separated from actual others (i.e., external separation). Synthesizing consciousness in people with ASD, those with schizophrenia, and those with the typical would diversify the relation between intentional consciousness and unconsciousness.

Here, we show that the trilemma of free will, determinism, and locality can help us understand the relation among intentional consciousness, unconsciousness, and actual others. Depending on the loss of one element in the trilemma, three possible types of consciousness can be obtained. One of the three types can reveal both distinction and integration of intentional consciousness and unconsciousness, which we call entanglement. Consciousness as an entanglement can bring about transformation and integration of passive action with active intention, and this can then achieve SoA and/or free will with determinism. As well as the statement, it was not possible to formulate the laws of quantum mechanics in a fully consistent way without reference to consciousness (Wigner 1967), consciousness is not possible to describe in a fully consistent way without reference to entanglement.

This paper is organized as follows. First, we explain the trilemma in Dummet's argument and show that it can be found in the brain. Second, we define the structure of consciousness through a combination of the presence or absence of the three elements in trilemma (free will, determinism, and locality). This results in three possible combinations: loss of free will (Type I consciousness corresponding to people with ASD), loss of determinism (Type II consciousness corresponding to people with schizophrenia), and loss of locality (Type III consciousness corresponding to typical people). In type I and type II consciousness, intentional consciousness and unconsciousness including readiness potential are either fused or separated. Intentional consciousness is entangled with unconsciousness only in 
type III consciousness. Third, we show that type I consciousness is consistent with various symptoms in ASD, for instance, weakened theory of mind and weakened embodiment. Type II consciousness is consistent with symptoms in schizophrenia, for instance, auditory hallucination, alien hand, self-other integration, and overestimation of SoA. Through investigation of fusion and separation, we clarify the significance of entanglement. Finally, we show that self-consciousness in the form of entanglement can implement SoA equipped with free will.

\section{Free Will, Determinism, and Locality in Dummet's Trilemma}

Dummett (1978) argued regarding trilemma comprising determinism, free will, and locality in the chief's dance for lion hunting success. The situation is explained as follows. In a village, to become social adults, boys must hunt a lion to show their courage. For two days, the boys travel to the hunting area. Then, they hunt lions for two days. Thereafter, they travel for two days to return to the village. Thus, it takes six days from their departure to arrival. Soon after the boys leave the village, the chief begins to dance for hunting success for six days. Dummett posed the following question: "Is it possible to argue for the chief because his last two days of dancing is done after the lion hunting and is nonsense?" According to Dummett, after four days, the lion hunt either succeeded or failed. If it failed, then the chief's dance was considered to be nonsensical. If it succeeded, then the chief's dance was considered to be redundant. Therefore, in any case, the chief's dance was considered to be irrational.

Some readers might now experience an incongruent feeling because they find no contradiction in the chief's dance, and they might behave like the chief. Indeed, imagine that dice is covered by an upside-down cup, and you have bet on an even number. Before the cup is turned upward, you pray for the even number, notwithstanding that the number is already determined underneath the cup. Since this attitude is the same as the chief's, you cannot comprehend Dummett's point. However, Dummett attempted to verify what makes the chief pray for a past event. Even if we frequently do the same thing, we do not know why we pray for what is already determined. Therefore, Dummet's question makes sense.

First, Dummett argued regarding fatalism, stating that a person's death is already determined. If your death is already determined, protecting yourself against death makes no sense. If it is determined that you will live, then protecting yourself against death is redundant. Therefore, protecting yourself against death is irrational. In fact, Dummett criticized fatalism with the following argument. A fatalist shifts from the statement "You will not die" to the statement "If you do not protect yourself against death, you will not die." However, these statements are not mutually relevant. Thus, the statement "If you do not protect yourself against your death, you will die" coexists with the previous statements. Thus, not protecting yourself against your death proves that you will both die and not die. That is inconsistent.

Dummett's argument seems difficult to comprehend. We attempt an explanation. Although a fatalist assumes that "You will not die," why you will not die remains undetermined, and multiple reasons can exist. In other words, although the goal is determined, paths toward the goal are not. If you do not take precautions against your death, you will not die. That is one possible path. The reason why you will not die could be your specific method of protecting yourself against death. That is another possible path. Thus, the second possible path implies that if you do not protect yourself against death, then you will 
die. Not taking precautions against your death proves both that you will die and that you will not die. As Dummett argued, fatalism can be denied.

Now, we return to the chief's dance. Note that we believe that we can change a future event, and we deny a fatalist who believes that a future event cannot be changed. In the chief's dance, however, our stance is reversed. The chief believes that he can change a past event, and he would criticize us for believing that a past event cannot be changed. The difference is only past and future. Dummett argued regarding the existence of determinism in which the cause determines the effect and then one can change the event. The difference between past and future is negligible.

The chief, as well as a fatalist, can actually say, "You shift from the statement The hunting is a success' to the statement If you do not dance, the hunting is a success.' " However, they are not mutually relevant. Thus, the statement "If you do not dance, the hunting is not successful" also coexists. Thus, no dance proves both the hunting's success and failure. That is inconsistent. Thus, "Dancing for the hunting's success is rational." Dummett stated that the chief's argument is the same as our argument against a fatalist.

In this argument, the logic is the same as ours. Even if hunting is successful, the reason for its success is not. Therefore, there can be multiple paths. If the chief does not dance, then the hunting is successful. That is one path. The reason for success could be only the chief's dance. That is another path, and it could imply that if the chief does not dance, then the hunting fails. Finally, no dance can prove both the hunting's success and failure. Note that here the hunting's result is identified by its report. Thus, one can argue multiple paths to the goal of reporting the hunt a success.

In this situation are three essential itemsfree will, determinism, and locality. Free will implies that the chief can start and stop dancing as he likes. Determinism implies the empirical law or the belief that the chief's dance can determine the hunt's result. Locality implies that the chief can know the hunt's result without affecting it before the boys return. In other words, locality implies that measuring objects (e.g., result of hunting) can be separated from an observer (e.g., the chief).

In this situation, Dummett argued that free will, determinism, and locality constitute a trilemma. Figure 1 shows a comparison between the chief's stance and Dummet's stance. Free will, determinism, and locality are indicated by thick arrows, where each stance's chosen items are indicated by yellow arrows and items not chosen are indicated by blank arrows. The chief has free will to dance, and he believes that his dance can determine the hunt's result. Thus, the arrows representing free will and determinism are indicated in yellow. Imagine that locality also holds in the chief's stance. Locality implies that the chief must dance where he knows the hunt's result. Assume that determinism holds. If the hunt succeeded, then the chief must dance even if he does not want to do so. Similarly, if the hunt failed, then the chief must not dance even if he wants to do so. There is no free will in this situation. Inversely, assume that free will holds. Whether the chief decides to dance or not, independent of the hunt's result, determinism does not hold. Thus, free will, determinism, and locality can constitute a trilemma. Finally, Dummett argued that the difference between Dummett (or modern people) and the chief is just a difference of culture. Both Dummett and the chief accept the trilemma; the chief relinquishes locality, and Dummett relinquishes determinism, as shown in Fig. 1. These two are possible parallel stances. No one can determine which is better, and thus, we cannot argue against the chief.

We previously showed that the free will theorem proposed in quantum physics (Conway and Kochen 2006, 2009) can contain three essential items: free will, determinism, and locality that constitute a trilemma. Such a trilemma can be found in the relation between intentional consciousness and readiness potential (Gunji et al. 2017). The next section 
Chief's stance

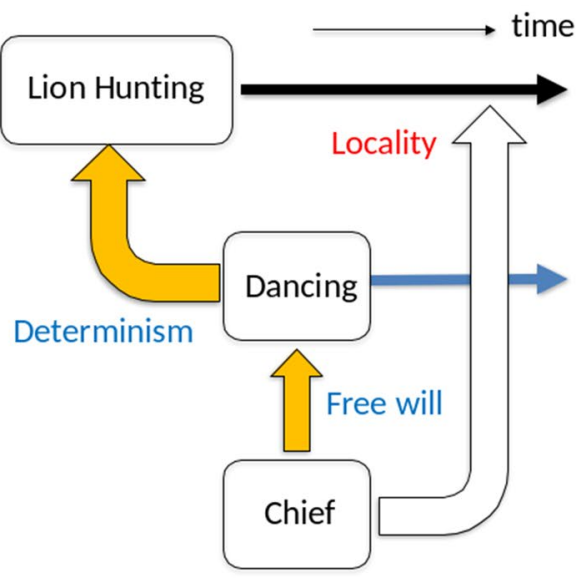

Dummet's stance

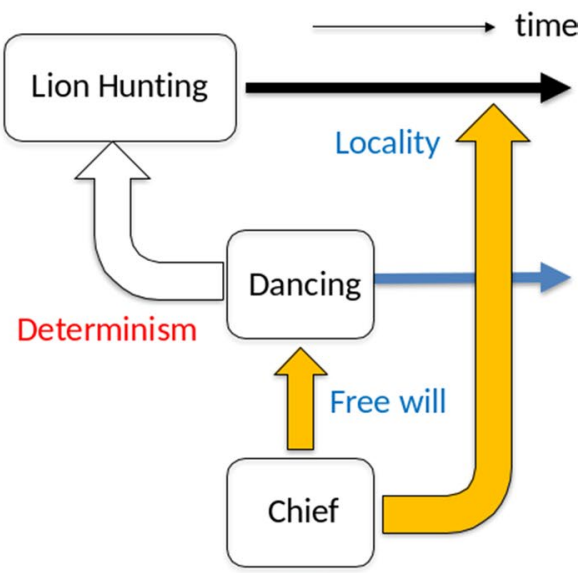

Fig. 1 Schematic diagram of the chief's dance for lion hunting success. One of the three trilemma elementsfree will, determinism, and localityis lost in each of the two stances. Absent elements are shown in red, and present elements are in blue. (Color figure online)

extends this idea using Dummet's argument to explain the mode of consciousness that includes typical symptoms of schizophrenia and ASD. Because three elements locality, determinism and free will constitutes tri-lemma (all three elements cannot co-exist due to the logical sense), one of three elements should be abandoned, as far as three elements can be found in real phenomena. Thus, the reason why one of three elements should be abandoned is not because of real properties but because of logical issue.

\section{Chief in the Brain}

Dummet's argument regarding the chief's dance can also be found in the brain. Libet et al. (1983) showed that the action of readiness potential precedes the action of intentional consciousness by $0.350 .5 \mathrm{~s}$. I intentionally think that "I will move my index finger" after readiness potential and/or subconsciousness is activated to move my index finger. In other words, intentional consciousness is passively forced to consider that "I will move my index finger" after my index finger's movement is determined. First, Libet claimed that there is no free will. Thereafter, he argued that there is free will if the intention to move the index finger suddenly changes to not move it. Libet argued that we can reject readiness potential through our free will (Libet 2004). However, free will in intentional consciousness never implies free will in "self" because self comprises intentional consciousness, readiness potential, and any other subconsciousness and unconsciousness (Dennett 2017). The next question that arises is how intentional consciousness, readiness potential, and any other subconsciousness could be integrated and/or entangled into a united consciousness. Here, we call such a united, integrated, or entangled consciousness self. First, we show that the structure of such self can be compared with that in Dummet's argument (Fig. 2). Recall the chief dancing for lion hunting's success as shown in Fig. 2's left diagram. The inner loop, self, represented by a broken line, implies bodily consciousness of the chief; the outer loop, society, implies the society to which the chief belongs. Since locality is lost, different 


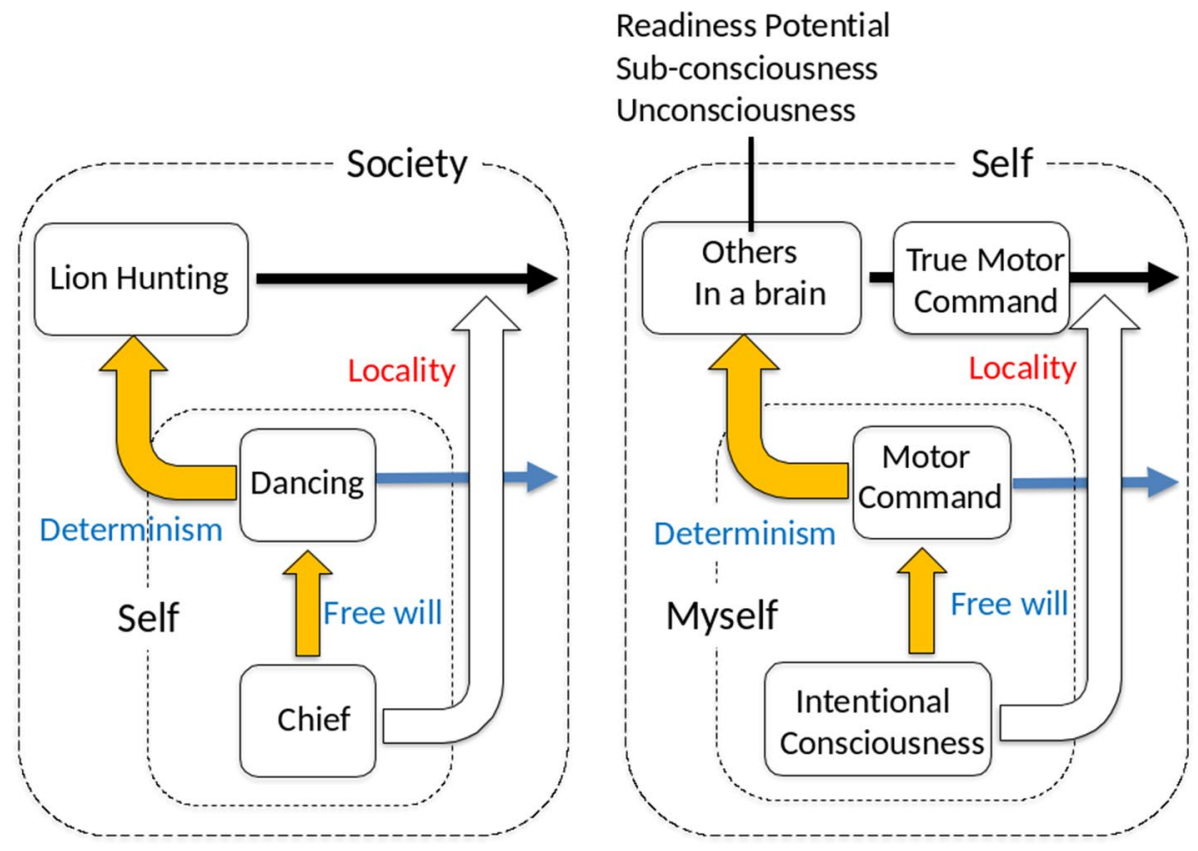

Fig. 2 Comparison of schematic diagram for the chief with one for consciousness

actions such as lion hunting and the chief's dance cannot be separated. Thus, the connection embedded in the chief's society is much stronger than those in the modern society. The chief who has free will and believes that he can determine hunting's result cannot measure that result (i.e., presence of determinism). This implies that the lion hunting's time development and that of the chief could be entangled.

The chief, lion hunting, and dancing can be replaced with intentional consciousness, readiness potential, and motor command in the brain, respectively. Similarly, the inner loop, self, in the left diagram is replaced by the inner loop, "myself," in the right diagram of Fig. 2. The outer loop of society in the left diagram is replaced by the outer loop of self in the right diagram. Intentional consciousness is believed to determine motor action and to have free will. However, true motor command driven by readiness potential precedes motor command driven by intentional consciousness. Clearly, intentional consciousness in the brain can be compared to the chief in Dummet's argument.

In Fig. 2's right diagram, intentional consciousness and motor command, which never influences motor action, could constitute myself. Any other information processing outside myself is represented here by "others in the brain" comprising readiness potential, subconsciousness, and any other unconsciousness. Like entanglement of the chief and lion hunting, myself and others in the brain could be entangled. In typical people, this entanglement can constitute self or consciousness. Since intentional consciousness follows readiness potential, any voluntary intention is passively forced by others in the brain. Despite such passivity, intentional consciousness can declare activity. Due to entanglement, passivity is assimilated with activity, and typical people have the sense of voluntary action and/ or sense of agency. Finally, we can conclude that loss of locality from the trilemma comprising locality, free will, and determinism can constitute typical people's consciousness. Either of readiness potential (RP) or intentional consciousness (IC) could not lead to free 
will and/or choice of action. The dynamic interaction between RP and IC can lead to the awareness of free will. It will be argued later.

With regard to the trilemma, there are three possible cases in which one item is abandoned. The first case (i.e., the chief or typical people) loses locality. The second case loses free will. The third case loses determinism. As mentioned previously, we assign the combination of either presence or absence of three items for the type of consciousness, which is inside the body. How can the combination of three items create the structure of consciousness or self? We propose a specific rule in which the three items' (myself, others in the brain, and self) roles are defined. In our model, self can comprise myself and others in the brain and can be surrounded by its own environment. The three items' roles are defined according to the function in the boundary among self, myself, and others in the brain. There are just two rules:

1 If locality is present, the boundary is either explicit or implicit. If locality is absent, the boundary is intermediate.

2 In our model of self, there are two boundaries, the inner boundary that is a margin of myself and the outer boundary that is a margin of self. Free will contributes only to the inner boundary, and determinism contributes only to the outer boundary.

These two rules are illustrated in Fig. 3a, in which the upper diagram shows rule (1). If locality is present, the boundary is explicit (solid thick line) or implicit (broken thin line) dependent on the boundary condition determined by the state (present or absent) of free will and determinism. The diagrams on the middle and bottom rows in Fig. 3a show rule (2), the function of free will and determinism. By combining rules (1) and (2), one can determine whether boundaries are explicit or implicit. If locality is present and free will is present, then the inner boundary is explicit. If locality is present and free will is absent, then the inner boundary is implicit. Because free will contributes to the inner boundary, it can result from rules (1) and (2). Similarly, if locality is present and determinism is present, then the outer boundary is explicit. If locality is present and determinism is absent, then the outer boundary is implicit. In contrast, if locality is absent, then the boundary is intermediate. Since the trilemma allows just three cases, the loss of locality implies the presence of both determinism and free will. Thus, both inner and outer boundaries must be intermediate; this can play an ambiguous role both inside and outside.

From rules (1) and (2), one can classify three kinds of consciousness, as shown in Fig. 3b. As mentioned before, one of the three items is absent, and this determines the combination of three items. In the first one, free will is lost, as shown in Fig. 3b's upper diagram. In this case, since locality is present, one of the two boundaries is explicit, and the other is implicit because of rule (1). Since free will is lost and determinism is present, the inner boundary is implicit (broken thin line) and the outer boundary is explicit (solid thick line) due to rule (2). In the second case, determinism is lost, as shown in Fig. 3b's middle diagram. This case also allows the presence of locality. Since free will is present and determinism is lost, the inner boundary is explicit (solid thick line) and the outer boundary is implicit (broken thin line) due to rule (2). In the third case, locality is lost. Due to rule (1), any boundary is intermediate. Since both free will and determinism are present, both inner and outer boundaries are intermediate, as shown in Fig. 3b's lower diagram.

In our model, except for diagrams shown in Fig. 3b, there is no other structure of consciousness. Clearly, asymmetric structure can be seen in terms of boundaries. The first boundary is a pair of implicit inner and explicit outer boundaries. The second is a pair of explicit 
A

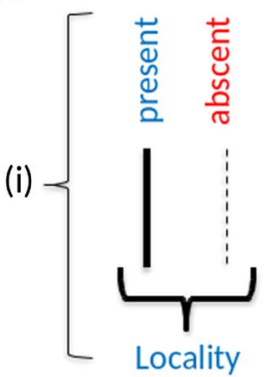

B

Locality
B

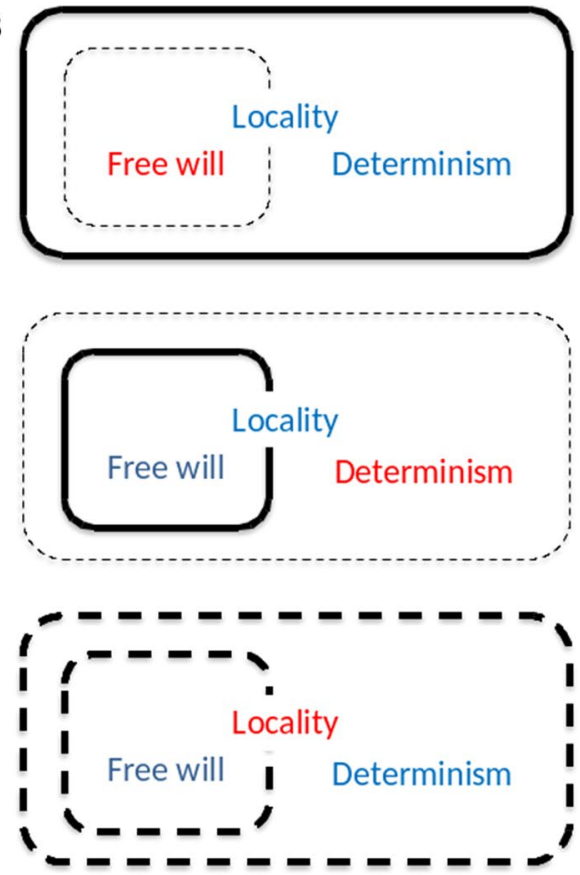

Fig. 3 a (1) Rules of locality. If locality is present (blue), the presence of other elements (blue; free will or determinism) generates an explicit boundary (thick solid line) and their absence (red) generates an implicit boundary (thin broken lines). If locality is absent (red), boundaries are intermediate (thick broken lines). (2) Free will is adapted to the inner boundary, and determinism is adapted to the outer boundary. b Combinations of the presence or absence of three elements leads to three possible types of consciousness if rules of $\mathbf{a}(1)$ and (2)are applied. (Color figure online)

inner and implicit outer boundaries. The first boundary implies fusion between intentional consciousness (myself) and others in the brain on the one hand and separation between self and actual others outside the body on the other hand. It implies no explicit other in the brain and that self is isolated from actual others. We believe that this kind of consciousness could be implemented in people with ASD. The second boundary implies separation between myself and others in the brain and fusion between self and actual others outside the body. This boundary implies explicit others in the brain and that there is self-other integration for actual others outside the body. This kind of consciousness could be implemented in people with schizophrenia. Shown in Fig. 3b's lower diagram, the third case shows both intermediate inner and outer boundaries, implying that intentional consciousness can balance with others in the brain and that self can balance with actual others. We believe that inner balance can constitute the embodiment of consciousness and that outer balance can constitute sociality. In the next section, we discuss this in greater detail. 


\section{Three Kinds of Consciousness}

Free will, determinism, and locality can play a role in appearing (i.e., explicit) or disappearing (i.e., implicit) boundaries between inside (e.g., intentional consciousness; myself) and outside (e.g., others in the brain). Free will cannot appear in enacting voluntary action if the agent that can take voluntary action never exists. Thus, free will in intentional consciousness can strengthen the inner boundary. Inversely, if free will is lost, the boundary between inside and outside is lost and inside (myself) and outside (others in the brain) are integrated and fused. Locality could strengthen separation from inside and outside so far as free will is present. Thus, the combination of locality and free will can explicitly separate inside (myself) from outside (others in the brain). Since intentional consciousness and others in the brain are integrated as unity because of loss of free will, locality cannot contribute to separate intentional consciousness from others in the brain. Instead, the combination of locality and determinism can contribute to separate self, comprising intentional consciousness and others in the brain, from outside because determinism can contribute to the outer boundary.

If locality is lost, intentional consciousness and others in the brain are entangled. Such an entanglement can entail the self, which can also be entangled with actual others outside the body. If locality exists in a trilemma, the combination of locality and free will enforce separation of intentional consciousness from others in the brain. The combination of locality and determinism enforces separation of self from actual others outside the body. Because trilemma holds that if one of three items is lost, there are three possible cases: the loss of free will, determinism, or locality. In our consciousness model, we distinguish entanglement from fusion. In entanglement of the inside and outside, the outside is accompanied with the inside but is hidden. By contrast, the fusion of the inside and outside could imply assimilation the inside with the outside. That can explain some misleading others in ASD and schizophrenic people. Here, we show that these three cases correspond to the consciousness of typical people, that of people with ASD, and that of people with schizophrenia.

First, we show that the combination in which free will and determinism remain and locality is lost (the left diagram of Fig. 4 could entail typical people's consciousness. In this case, free will contained in intentional consciousness can act on others in the brain, and intentional consciousness itself believes that it can determine other processes in the brain. Clearly, readiness potential contained in others in the brain precede voluntary intention appearing in intentional consciousness. In this sense, intentional consciousness is passively forced to consider voluntary intention, while intentional consciousness can believe that it actively determines her/his own decision making. Due to the entanglement of intentional consciousness with others in the brain, intentional consciousness can assimilate the active mode with the passive mode of actions

The right diagram of Fig. 4 shows that the entanglement of myself and others in the brain could also entangle actual others outside the body. Entanglement is nothing but loss of locality. While different information processing in the brain, such as myself (intentional consciousness) and others in the brain can be independently distinguished, different information processing can be assimilated and mutually identified. This is called entanglement. The inner solid loop (i.e., inner boundary) represents myself comprising intentional consciousness and motor command triggered by intentional consciousness, in which motor command is abbreviated. Note that true motor command is triggered by readiness potential contained in others in the brain, as shown in Fig. 2. 


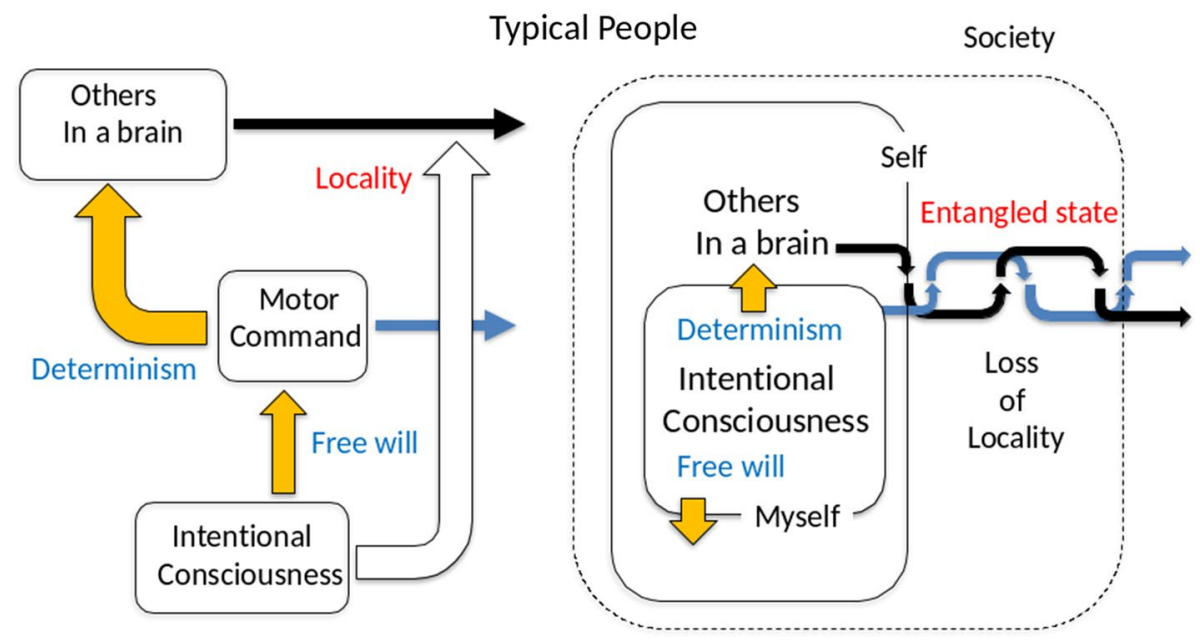

Fig. 4 Structure of consciousness corresponding to typical people. It results from trilemma with loss of locality. Intentional consciousness or myself is entangled with others in the brain

Entanglement is the only way by which intentional consciousness can claim free will and determinism. In other words, intentional consciousness in typical people can balance the relation between inside (myself; intentional consciousness) and outside (others in the brain). On the one hand, the inside and outside can be distinguished; on the other hand, the inside can communicate and assimilate with the outside. Due to loss of locality and rule (1), explained above, both inner and outer boundaries must be intermediate. This implies that self also balances with actual others outside the body and results in adequate communication between self and actual others. There is no self-other integration in people with schizophrenia and no excess isolation from others in people with ASD. Such an intermediate and adequate balancing between self and actual others could be achieved only by the entanglement of intentional consciousness and others in the brain. This can be immediately related to sociality. Second, we show that the combination in which locality and determinism remain and free will is abandoned (Fig. 5, left diagram) could entail consciousness of the people with ASD. As mentioned previously, since free will that can contribute to the inner boundary is lost, the boundary between intentional consciousness and others in the brain is weakened or disappears in the presence of locality. This could result in strong fusion of myself and others in the brain as unity, as shown in Fig. 5's right diagram. The presence of determinism, which can contribute to the outer boundary, can strengthen the boundary between self and actual others outside the body in the presence of locality. In other words, self could be isolated from actual others, and people with ASD find it difficult to communicate with actual others outside the body.

This situation seems consistent with the typical behavior of people with ASD. While ASD patients seem closed against other people outside the self, voices of other people could just sound like undifferentiated noise. The patient cannot recognize the external world and/or others until what others call "voice" can be recognized not as noise but as voice. In most cases, such voice could arise from the mother. Thus, if a 9 months old infant loses the chance to recognize the first voice from the mother, the infant could become a person with severe ASD (Utsumi 2015). If a mother's voice reaches an infant's 


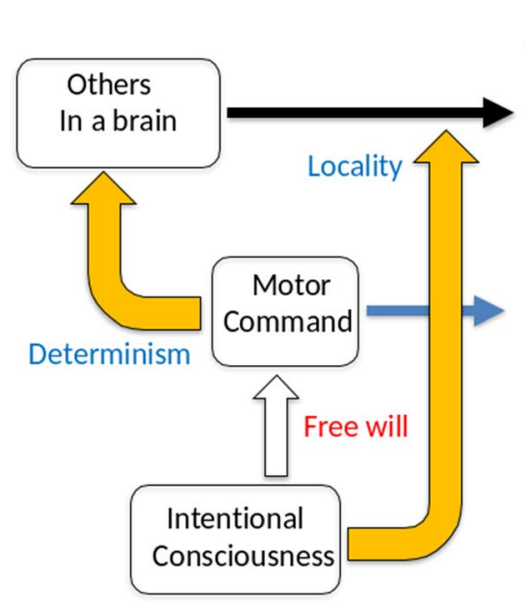

ASD

Fig. 5 Structure of consciousness corresponding to ASD. It results from trilemma with loss of free will. Intentional consciousness or myself is fused with others in the brain and that generates self-separated from actual others

mind, it leaves its trace there. In typical people, self grows from the core of that trace. Thus, if a mother's voice never reaches an infant's mind, then self may not develop.

Our model is consistent with Utsumi's explanation. Self cannot be divided into myself and others in the brain until the infant recognizes the mother's voice. In other words, the mother's voice can trigger the brain to split the self into myself and others, with an intermediate boundary. Her voice can bring about the entanglement of myself and others in the brain, not a fusion of them. In our model, determinism exists and strengthens the outer boundary, which can separate self from others outside the body. As a result, asymmetry between inner and outer boundaries can occur. How does the fusion of myself and others in the brain look? Is it a collection of isolated elements? In the self of people with ASD, components of self, such as myself and others in the brain, are tightly connected to each other. This implies the presence of determinism and results in excess fusion of parts and whole or in the impossibility of distinguishing parts. This could be impaired agency and/or ownership of bodily image.

Third, we show the combination in which locality and free will remain and determinism is abandoned (Fig. 6, left diagram). In the presence of locality, the presence of fee will strengthens the inner boundary (i.e., explicit) and absence of determinism weakens the outer boundary (i.e., implicit). Self can be divided into some compartments that can be separated because determinism is lost and components in the self cannot be controlled and/ or cannot be determined by other components. People with schizophrenia feel as if there were others in the brain whom he/she cannot control alone. Thus, people with schizophrenia can feel as if others' thoughts were inserted (thought insertion) and others' voices were heard in her/his brain (auditory hallucination). Here, such a situation is called self-other separation. Asymmetry between inner explicit and outer implicit boundaries can explain self-other integration, an attribute of schizophrenia. Since the outer boundary between self and actual others outside the body could be implicit or weakened, people with schizophrenia can assimilate themselves with others. People with schizophrenia who are in front of emotional others can be emotional. Self-other integration and self-other separation are both sides of the same coin in our model's context. 


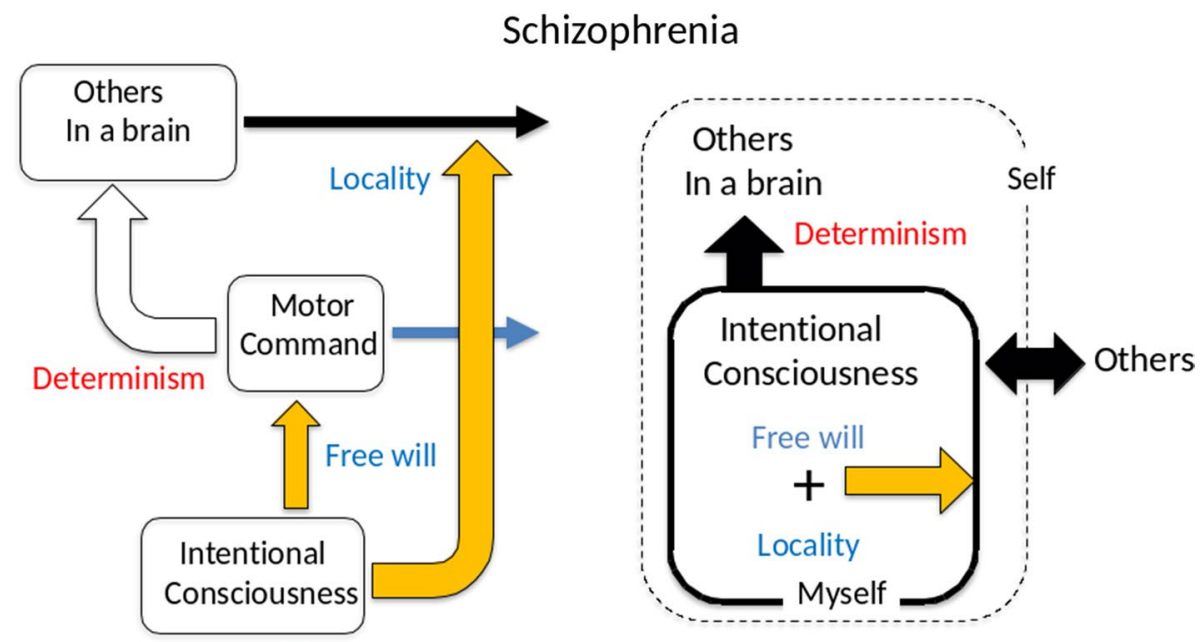

Fig. 6 Structure of consciousness corresponding to schizophrenia. It results from trilemma with loss of determinism. Intentional consciousness or myself is completely separated from others in the brain and that generates self-fused with actual others

\section{Entanglement Versus ASD and Schizophrenia}

\subsection{General Classification of Consciousness}

In the previous section, we described how three kinds of consciousness can be explained through the trilemma paradigm. We also sketched how three kinds of consciousness can be related to typical people, people with ASD, and people with schizophrenia. In this section, we discuss in detail how characteristic symptoms of people with ASD and schizophrenia are related to the symptoms discussed in our model. Recently, several experiments on SoA and $\mathrm{SoO}$ of body were conducted with people with ASD and those with schizophrenia. Self-concept and/or bodily consciousness in people with ASD and with schizophrenia were reported to be impaired. Here, we show how impaired function of SoA and SoO in people with ASD differs from that in people with schizophrenia. From these considerations, the significance of entanglement in typical people can be explained.

Figure 7 shows the comparison of three types of consciousness that arise from the trilemma. We refer to consciousness resulting from the combination without free will as type I consciousness, that without determinism as type II consciousness, and that without locality as type III consciousness. The boundary's property is classified into fusion, separation, and entanglement with respect to functions operating inside and outside. Seeing the asymmetric structure of fusion and separation is easy in type I and type II consciousness. By focusing on asymmetric structure within a boundary term, one can explain impaired self-consciousness and bodily consciousness in ASD and auditory hallucination, thought insertion, and self-other integration in schizophrenia. Intermediate boundary which is neither fusion nor separation can reveal entanglement of the self and others. There are some attempts to implement the others in a brain in logical fashion (Lefebvre 1987).

Three cases of refusal for the trilemma (i.e., loss of free will, determinism, or locality) can reveal three types of bodily consciousness corresponding to three kinds of consciousness (Fig. 8. Properties of boundary, fusion, separation, and entanglement can entail 


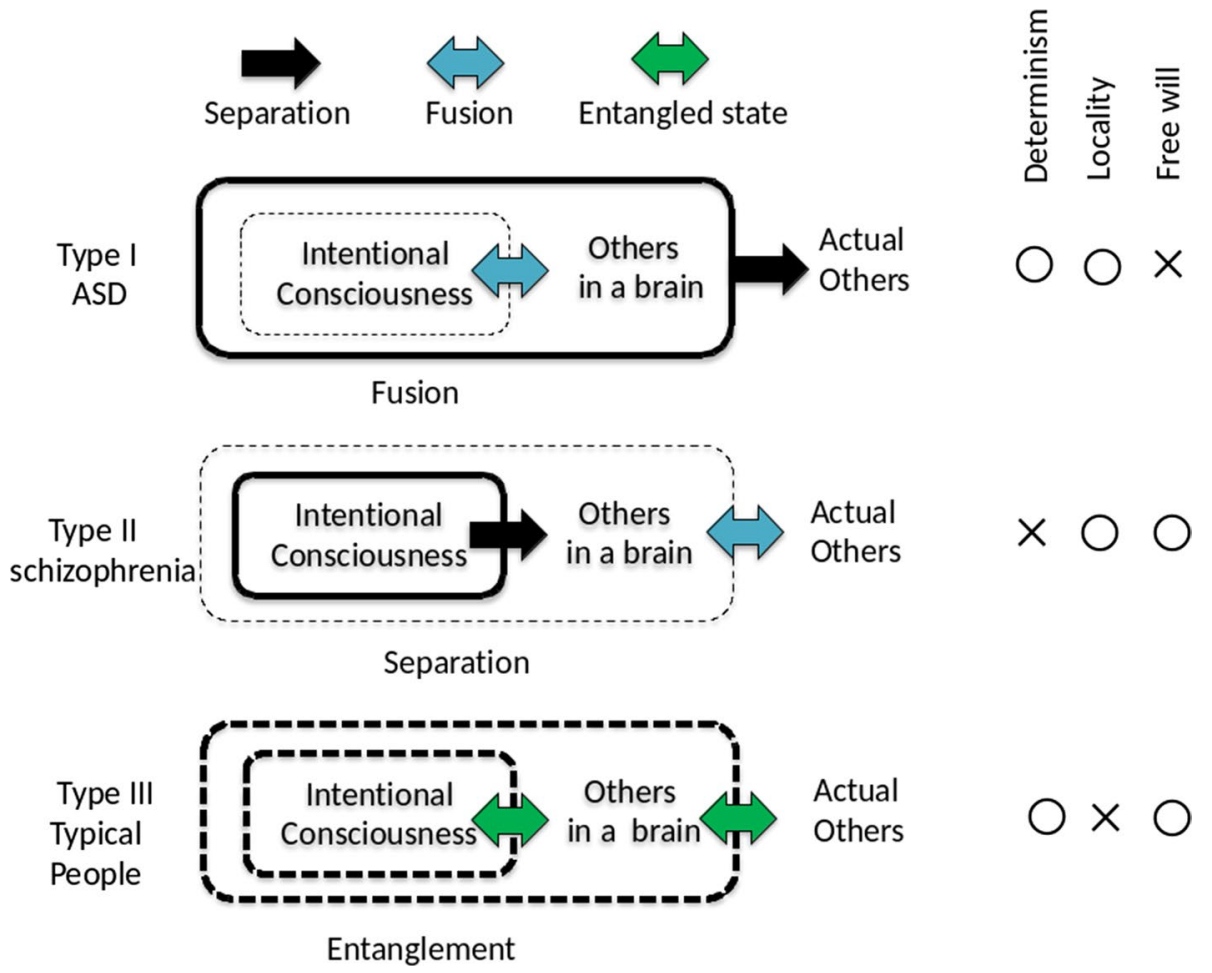

Fig. 7 Classification of type I, II, and III consciousness with respect to boundary properties. A present element is represented by a circle and by a cross otherwise. The combination of elements and rules (1) and (2) produces three types of consciousness

function or dysfunction of acquisition of the SoA and $\mathrm{SoO}$ of one's body. SoA is defined as the sense in which one causes the motion of one's body, and SoO is defined as the sense of one's body belonging to oneself (Gallagher 2000, 2007; Haggard et al. 2002a, b; Tsakiris et al. 2006; Paillard 1999; Kammers et al. 2009; Cunningham et al. 2008). Classically, SoO is studied using the rubber hand illusion (Botvinick and Cohen 1998; Botvinick 2004; Costantini and Haggard 2007; Ehrsson et al. 2004; Farne et al. 2000; Longo et al. 2008; Tsakiris and Haggard 2005), and SoA is studied using a robot or virtual reality that can be moved synchronously with the participant's own body (Farrer and Frith 2002; Frith 2005; Ismail and Shimada 2016). Implicitly, the latent intensity of SoA and SoO is estimated in questionnaire results. Explicitly, the intensity of SoO is measured by proprioceptive drift and that of SoA is measured by intentional binding (Haggard and Clark 2003; Haggard et al. 2002a, b; Haggard and Cole 2007; Moore and Haggard 2008). Intentional binding can reveal that the time interval between the participant's voluntary action and the following event could be perceived as shorter than the actual time interval (Moore and Obhi 2012; Moore et al. 2013). Although SoA and SoO behave interdependently, they might actually be independent, as shown through the experiment with the moving rubber hand (Kalckert and Ehrsson 2014; Louzolo et al. 2015). Whether they are interrelated remains unclear.

In our model, both SoA and SoO can hold only if entanglement between inside and outside can be achieved. In typical people, although voluntary intention to move a finger 


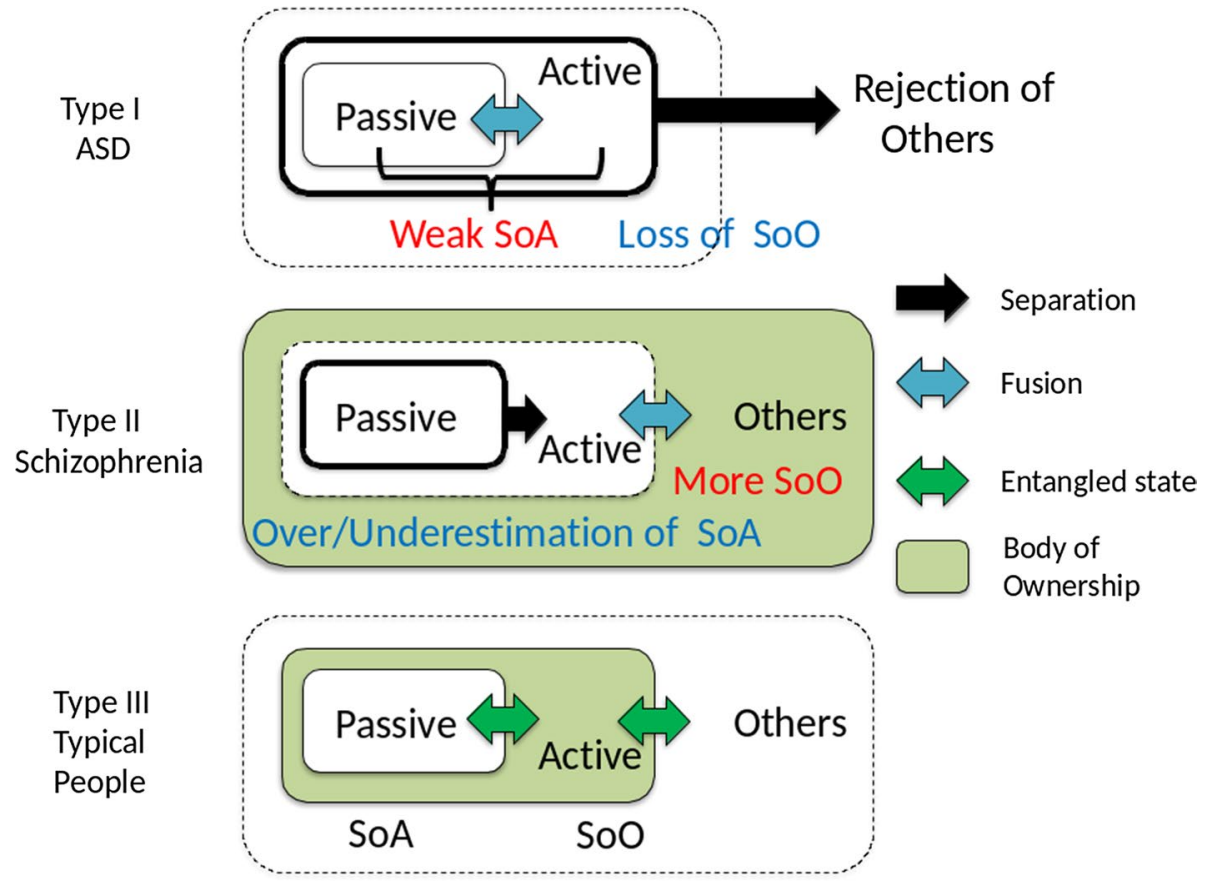

Fig. 8 Schematic diagram of bodily consciousness (embodiment) obtained from type I (top), type II (middle), and type III (bottom). Type I embodiment reveals that rejection of actual others cannot generate embodiment featuring others. Type II reveals excess of embodiment because of fusion of self and actual others. Type III reveals adequate embodiment due to entanglement

appears in myself (intentional consciousness) as passively enforced by others in the brain (readiness potential), myself claims that such action appears actively. Such replacement of the passive with the active aspect can lead to SoA. There is ambiguity between distinction and synthesis of information processing in myself and that in others in the brain. Because readiness potential precedes the firing of intentional consciousness by approximately $350 \mathrm{~ms}$, SoA cannot be achieved until the passive aspect is replaced with the preceding active aspect. This is nothing but the entanglement of two kinds of information processing (Fig. 8), bottom diagram).

Type I consciousness has no entanglement in the inner boundary. Others in the brain is fused with myself. Thus, before assimilating myself with others in the brain, myself cannot be distinguished from others in the brain. This suggests that people with ASD cannot feel the same SoA as typical people. In type II consciousness, others in the brain is separated from myself. There is no entanglement. Thus, no identifying preceding readiness potential (in others in the brain) with intentional consciousness (myself) can weaken or lose SoA.

Entanglement in the outer boundary can play an essential role in SoO in type III consciousness. Actual others can generate and enhance bodily consciousness accompanied with SoO. Psychotherapist Sakaki (2001) clearly illustrated bodily consciousness with SoO. When others scratch typical people on the back and ask, "Right here?" the typical person can reply "That's where it itches." The typical person can indicate the itchy point with her/his own finger and say, "here." The identical itchy point on the back is called both 
"that point" and "this point." "This" is used in the egocentric perspective and "that" is used in the allocentric perspective via others. That is embodied flesh body with SoO characteristic to the ambiguity of egocentric and allocentric perspectives. In type III consciousness, clearly, the ambiguity of egocentric and allocentric perspectives can be implemented by the entanglement of the outer boundary. Sakaki (2001) argued that children with ASD cannot use both this and that for the same itchy point. They use only the egocentric view and this. According to Sakaki (2001), the flesh bodies of children with ASD are not embodied.

In contrast, type I consciousness generates a separate outer boundary between self and actual others. It explains that children with ASD cannot apply the ambiguity of egocentric and allocentric perspectives to their own bodies. Type II consciousness integrates self with actual others outside the self. While these considerations suggest that dysfunctions of SoA and $\mathrm{SoO}$ in people with ASD superficially resemble those in people with schizophrenia, the essential cause of the dysfunctions differs.

\subsection{Comparison of the Consciousness of ASD with Type I Consciousness}

\subsubsection{Self-Reference}

Since ASD is defined by impairments in communication and iterative behavior, many researchers have investigated how people with ASD generate social and behavioral deficits and impairments in perception and cognition (Kaiser and Shiffrar 2009). Children with ASD are reported to show weak central coherence, characterized by the loss of specific context as a whole (Happé and Frith 1996). Theory of mind can be diminished in children with ASD (Baron-Cohen 1995; Baron-Cohen et al. 1985). Classically, malfunction of communication in people with ASD has been explained by an extreme form of egocentrism (Bleuler and Bleuler 1986) and, recently, by a complete absence of sense of self (Frith 2003).

Autobiographical memory can emerge and be maintained by a self-referential process, and memory can make the sense of self dynamic and robust (Conway and Pleydell-Pearce 2000; Wang 2001). This sense of self can bring about an essential distinction between self and others and reveal the distinction of sense referring to the self and that referring to others. Memorization and recollection can be achieved more accurately related to self rather than to others based on the distinction of self and others (Rogers et al. 1977; Cunningham et al. 2008). This tendency is achieved even for 4-year-old to 6-year-old children (Cunningham et al. 2013).

Williams (2010) distinguished psychological self from physical self to explain the concept of the self and to argue that the psychological aspect might be impaired in children with ASD (Williams and Happé 2010; Uddin 2011). Evidence showing intact physical self in ASD has accumulated. Children with ASD can display mirror-self recognition (Ferrari and Matthews 1983), can recognize delayed video images of themselves (Lind and Bowler 2009), and can discriminate other and self-caused changes (Grainger et al. 2014b). In contrast, much evidence shows that psychological self is diminished, such as alexithymia (Hill et al. 2004) confusing the first and the second person (Lee et al. 1994); failure of various cognitive tasks (Grainger et al. 2014a). Williams and Happé (2009) and Williams and Happé (2010) show that children with ASD are not confused to find a concealed picture.

In spite of accumulation of evidence, impaired psychological self could result from the failure of distinction between self and others, and/or the dysfunction of self-reference is not clear. Such difficulty can be explained by our model, which is type I consciousness. 
Physical self and psychological self can be compared to self and the complex comprising self and actual others in our model. In our model, type III consciousness corresponds to typical people with the intermediate boundary. Thus, while self and actual others can be discriminated, self and actual others can also constitute the complex comprising self and actual others. Only in this situation can people have psychological self. People can have both egocentric and allocentric views via the psychological self (Riva and Gaudio 2012; Riva 2012). In contrast, type I consciousness that could underlie the consciousness of people with ASD is characteristic of implicit inner boundary and explicit outer boundary. Especially, the explicit outer boundary can separate self from actual others and also refuse actual others, while people with ASD have strong curiosity for others because the self is fused with others in a brain. Thus, type I consciousness cannot constitute the complex corresponding to the psychological self. Although type I consciousness can discriminate self from actual others, that is an excess form of discrimination. Thus, in comparing people with ASD to the type I consciousness, people with ASD can explicitly discriminate physical self from others but cannot distinguish psychological self from others. Because the psychological self can contain some kinds of others, the aspect of others is implicitly contained and is explicitly separated from self. In considering type I consciousness, one can expect that such ambiguity of others cannot be grasped by people with ASD.

Ambiguity of others' aspect plays an essential role in self-reference. While self is discriminated from others regarded as "objects" outside the self, self must also be regarded as object since it is referred to by the self itself. In this sense, self and others are both sides of the same coin. However, it is very hard for people with ASD suggested by type I consciousness to perceive such ambiguity. Thus, genuine sense of self-reference cannot function in people with ASD in the sense of type I consciousness.

\subsubsection{Theory of Mind}

The complex of self and others is immediately related to perception and comprehension of others. Understanding others is regarded as understanding others' intention independent of the real world (Dennett 1989), and understanding others' intention could result from having a theory of mind (Premack and Woodruff 1978). False-belief tasks such as the SallyAnne test are proposed to discriminate whether patients have theory of mind (Baron-Cohen et al. 1985) because understanding another's intention is to solve the false-belief task. The underlying mechanism of understanding others' mental states is proposed in two ways: theory-theory and simulation theory (Carruthers 1996). Theory-theory claims that the intrinsic knowledge for understanding others is automatically acquired during development in a society, including social norm and/or social beliefs (Gopnik and Wellman 1992; Perner and Howes 1992). In this sense, understanding others is discriminated from understanding self. Simulation theory claims that understanding others is just mimicking others' behaviors and/or states (Gallese and Goldman 1998; Goldman 2009), and thus, understanding others is considered to be the same as understanding self. Although long debates have occurred between theory-theory and simulation theory, simulation theory recently predominates because it is thought to be supported by mirror neurons (Gallese et al. 1996; Rizzolatti et al. 1996; Gallese 2003).

Children with ASD do not employ theory of mind and that suggests their difficulty in understanding others' intentions. Although various explanations for this are proposed (Baron-Cohen 1991; Leslie 1991; Hobson 1995; Dapretto et al. 2006), they are based on the assumption of a strict distinction between self and others and between a certain level 
of information processing and a higher level of information processing. As for mirror neurons, such a strict distinction was originally observed; however, recently, mirror neurons are reported to be fired not only when subjects are grasping for an object themselves but also when they observe others grasping for an object (Gallese et al. 1996; Rizzolatti et al. 1996). Recent research suggests that although mirror neurons are not fired when an identical motor representation occurs, they are fired when the identical goal of different motor representation occurs (Bonini et al. 2013; Ocampo and Kritikos 2011). Also reported is that mirror neurons are immediately relevant for understanding emotion and anticipation (Carr et al. 2003; Iacoboni 2009). This implies that a lower level of information processing, such as motor representation, can be adequately discriminated and accompanied (partly identified) with a higher level of information processing, such as evaluation of representation and emotion influenced by the evaluation. Ambiguity of discrimination and identification is hidden in the function of mirror neurons.

Adequate discrimination and identification of different level information is the key notion in the intermediate boundary or entanglement in type III consciousness. Entanglement of myself and others in the brain can constitute the complex comprising self and actual others. Myself implies the focal level of information processing, and others in the brain implies information processing surrounding the focal and influenced by the focal level. If myself is compared to the motor representation, others in the brain are compared to various informational processes, including estimation of representation and emotion. Therefore, entanglement of myself and others in the brain can be consistent with recent evidence of mirror neurons.

In type I consciousness, fusion of myself and others in the brain can entail separation of self and actual others. This results in dysfunction of the complex corresponding to psychological self. In this sense, simple conflict between theory-theory and simulation theory on mirror neurons is to be abandoned because simulation theory focuses on only temporal immediate information processing (i.e., discrimination of the information processing level), and theory-theory focuses on only synthesized wholeness of information processing (i.e., identification of various levels of information processing). Recent studies regarding mirror neurons support both theories and suggest the entanglement of self and actual others and of myself and others in the brain.

Type I consciousness implies not impaired comprehension of others but impaired generation of the complex of self and actual others. In other words, self fails to be accompanied by others and/or the external world. Such openness implies intentionality to the external world or others, and the function of the subject's own perspective (i.e., context). This suggests that no employment of theory of mind in people with ASD can result from the impossibility of taking any perspective, not from the impossibility of taking another's perspective. Type I consciousness suggests that people with ASD cannot take their own perspective but just a representation of the objective external world. Thus, unlike typical people, it is very hard for those with ASD to have their own intentionality.

\subsubsection{Loss of SoO}

While recently some studies have investigated SoA in people with ASD, their results conflict. In manipulating visual feedback of a cursor in a monitor, SoA was not reduced more in subjects with ASD than in the control population (David et al. 2008). However, SoA was reduced in people with ASD in the task with a gaze-contingent lens in a social context (Grynszpan et al. 2012). The conflict in terms of experimental results might result from the 
ambiguity of the SoA concept itself since SoA contains various meanings such as judgment of agency (JoA) and feeling of agency (FoA) (Synofzik et al. 2008). Reasons for conflict in SoA in people with ASD remain unclear.

Motor behavior in people with ASD is commonly reported as diminished, including motor planning, monitoring, and prediction (Grynszpan et al. 2012; Martineau et al. 2004; Nazarali et al. 2009; Gowen and Hamilton 2013). In controlling a task, children with ASD were able to use a feedback process but not a feed-forward process (Schmitz et al. 2003). Estimation of EEG activity in people with ASD suggests that abnormal activity of a supplementary motor area can contribute to preparation and selection of movements (Martineau et al. 2004; Enticott et al. 2009; Rinehart et al. 2006) and is immediately relevant for intentional binding (Moore et al. 2010; Kühn et al. 2013). Recently obtained evidence shows reduced intentional binding in individuals with ASD (Sperduti et al. 2014).

As for SoO, individuals with ASD show smaller proprioceptive drift in the rubber hand illusion (Paton et al. 2012); this suggests that individuals with ASD have little acquisition of SoO in the rubber hand. While ASD is known to include a disorder of cognitive empathy (Rogers et al. 2007), strong empathy is correlated with strong SoO in the rubber hand illusion (Asai et al. 2011). Recent studies have shown that oxytocin, a hormone acting as a neurotransmitter, modulates social cognition and empathy (Kosfeld et al. 2005; Baribeau and Anagnostou 2015; Wigton et al. 2015), that several variants in oxytocin were found with ASD (Lopatina et al. 2013), and that oxytocin can improve social cognition (Cochran et al. 2013). A recent experimental study revealed that high oxytocin concentration is associated with SoO (Ide and Wada 2017).

In our model, type I consciousness reveals the inseparability of myself and others in the brain. Thus, myself cannot manipulate the relation between readiness potential and intentional consciousness. Before the assimilation of preceding active readiness potential with passive intentional consciousness, the two cannot be separated. Thus, an efferent copy of intention toward the motor system can be neither found nor manipulated and the feed-forward process cannot be realized in self. Motor representation, its evaluation, and its affective emotion cannot be separated when fusion of myself and others in the brain is strengthened, and both JoA and FoA are diminished. If such fusion is weakened, to some extent, either JoA or FoA is diminished. Depending on fusion's extent, impaired SoA can appear in several ways. This is consistent with the actual tendency in people with ASD.

Clearly, SoO can be associated with sociality or psychological self (Baribeau and Anagnostou 2015; Wigton et al. 2015). In our model, psychological self is implemented by the complex comprising self and actual others. The complex can appear only when the outer boundary is intermediate in type III consciousness. type I consciousness cannot generate intermediate outer boundary and keep actual others away. Thus, the embodiment of the flesh body cannot be achieved, and $\mathrm{SoO}$ is diminished.

\subsection{Comparison of the Consciousness of Schizophrenia with Type II Consciousness}

\subsubsection{Integration of Others and Self}

Schizophrenia is characterized by self-disturbance, that is, impaired balance between self and others. The individual with schizophrenia finds her/himself in others and finds others in her/himself (Bleuler and Bleuler 1986; Hemsley 1998; Mishara et al. 2013; Sass and Parnas 2003; van der Weiden et al. 2015). Although impaired balance can reveal both finding her/himself in others and finding others in her/himself, our model claims that they can 
be discriminated and that finding her/himself in others, such as through emotional cognition, could result from the fusion of outside and inside of outer boundary. Finding others in her/himself, such as in thought insertion and auditory hallucination, could result from the separation of outside and inside of the inner boundary. Our model of consciousness is characteristic of type II consciousness.

While positive symptoms are characteristic of some symptoms added to typical consciousness, for example, delusion of control and delusion of reference, negative symptoms show absence of some characteristics of typical consciousness, for example, affective flattening and anhedonia (Sass and Parnas 2003; Sass 2014). Both positive and negative symptoms can also be explained in the context of type II consciousness. In delusion of reference, the individual with schizophrenia feels as if an event caused by others was caused by her/ himself (Synofzik et al. 2013). This is typical integration of others and self and can be explained by the fusion of self and actual others. Affective flattening can be explained by the separation of myself and others in the brain. In our model of consciousness, they are discriminated in terms of the causal mechanism.

First, we argue regarding the symptoms that can be explained by the fusion of self and actual others. In the model of type II consciousness, loss of determinism entails implicit outer boundary between self and actual others and that brings about the fusion of self and actual others (Fig. 7, middle diagram). Because of this fusion, the action caused by others is felt as if they were caused by the individual with schizophrenia (Gould 1948; Green and Kinsbourne 1990; Van Der Gaag 2006). Indeed, it entails emotional cognition (Montag et al. 2007).

Importantly, the establishment of separated self and others precedes fusion process. Thus, although people with schizophrenia can discriminate her/himself from others, impaired identification and/or synthesis could occur (Abu-Akel and Shamay-Tsoory 2013). People with schizophrenia could suffer from difficulty in constructing the relation between self and others (Pinkham et al. 2007). While the reason for difficulty has been explained by the loss of sociality (Fett et al. 2011), the difference between ADS and schizophrenia with respect to the reason for integration of self and others has not been clarified. As mentioned in the section on type I consciousness, sociality associates with mirror neurons that can be relevant not only for motor representation but also for its evaluation and influenced emotion. Firing of mirror neurons occurs less in people with schizophrenia compared with typical people (Mehta et al. 2014). Here, to clarify this, we introduce the distinction between prediction and anticipation. Prediction implies just computing motor representation in advance, and anticipation implies prediction accompanied by its context. The context includes the significance and the goal of motor representation and/or emotion. In this sense, mirror neurons in normal condition could be employed for anticipation rather than for prediction (Iacoboni 2009). In contrast, impaired mirror neurons in people with schizophrenia could be employed not for anticipation but for prediction. Because of the loss of context, distinguishing self from others is difficult. The individual with schizophrenia, therefore, introduces knowledge such as social norms and beliefs in addition to prediction. While recruitment of a variety of social knowledge could help the individual with schizophrenia to infer others, such attempts at inference are very hard, and most result in failure. Indeed, this entails the integration of self and others with respect to emotion (Corbera et al. 2013; Decety and Lamm 2011; McCormick et al. 2012; Smith et al. 2014).

In our model, type II consciousness, loss of determinism, and presence of free will and locality can individualize myself and others in the brain. A component of self is isolated from each other. This implies that the relationship and context surrounding components cannot be manipulated although each state of the component can be computed. The 
context, significance, and influenced emotion of the future state are lost. The situation can correspond to impaired mirror neurons' activity. In other words, type II consciousness is employed not for anticipation but for prediction. Since loss of determinism also entails implicit outer boundary, self and actual others are fused into unity. This is also supported by self comprising isolated components because such self has no ability to discriminate self from actual others.

Compared to type I consciousness, the difference is clear. type I consciousness refuses actual others and keeps them away. It cannot identify others with intentionality. In contrast, type II consciousness identifies others and discriminates self from actual others, but this fails due to the underlying mechanism. Through correspondence between type I and ASD and between type II and schizophrenia, one can say that the difficulty of social communication in people with ASD differs from that in people with schizophrenia.

\subsubsection{Others in Myself}

The concept of delusion of reference sounds misleading. It assumes the preexistence of self who can determine a reference to an indicated object. This is the immune principle of consciousness (Gallagher 2000). In our model, such a typical self can be established only when inside and outside can be entangled as in type III consciousness. Type I consciousness can reveal an excess form of integration of myself and others in the brain as fusion. Type II consciousness cannot achieve self in which myself and others in the brain can be synthesized. In other words, components in self are split in type II consciousness. In this context, we discriminate finding others in myself from myself in others. Myself in others implies integration of myself and others because both myself and others are established. Others in myself implies that some parts of myself are isolated from the core of myself (e.g., intentional consciousness), and that implies an imperfect myself. In our model, others in myself can be expressed as "others in the brain that are isolated from myself and exist in self." Others in the brain in self is a typical attribute of schizophrenia (Asai et al. 2011; Jardri et al. 2009, 2011). Voice shouted from her/himself can be considered as another's voice (Green and Kinsbourne 1990; Van Der Gaag 2006), and hand movements caused by her/himself can be considered as caused by an alien (Frith 2005). In type II consciousness, these symptoms can be explained by explicit inner boundary and/or separation of myself and others in the brain. Our consciousness model's significance is proposing asymmetric structure of inner and outer boundaries. In this asymmetry, others in myself (others in the brain in self) is discriminated from myself in others (integration of self and actual others). Delusion of reference is replaced by the asymmetry between fusion and separation. Recall that this asymmetry is also found in type I consciousness, where the relation of fusion and separation is reversed. This implies an intrinsic difference between ASD and schizophrenia.

\subsubsection{Over and Underestimation of SoA}

Since simulation theory as the underlying mechanism of theory of mind was supported by mirror neurons, goal orientation and emotion accompanied prediction (here called anticipation) has been investigated in individuals with schizophrenia. As mentioned before, theory of mind works abnormally in individuals with schizophrenia (Brüne 2005; Derntl et al. 2009; Green et al. 2011; Varcin et al. 2010). The underlying mechanism of impaired working of theory of mind could not be dysfunction of simulation itself of motor representation 
(McCormick et al. 2012; Thakkar et al. 2014), but erroneous decision of self and others in terms of prediction accompanied by its estimation and emotion (Thakkar et al. 2014). This entails excess assimilation of self and others because discrimination of self and others is attempted "logically" without emotion and fails (Corbera et al. 2013; Montag et al. 2007; Smith et al. 2014).

As mentioned in section 5-2 on type I consciousness, we argue that no conflict exists between theory-theory and simulation theory. This implies that normal mirror neurons can be employed for both simulation (i.e., prediction) and theory-theory (i.e., acquiring social context and/or social norms through inference and emotion). Although people with schizophrenia can predict others' future states, they cannot accurately predict others' mental states (Green et al. 2008; Kring and Elis 2013). Since they cannot infer the atmosphere of communication, they either over or underestimate the other's state in mimicry of yet others (Kring et al. 1999; Varcin et al. 2010). Such a symptom is consistent with our explanation based on type II consciousness. Because in type II consciousness, components of self are isolated, one can predict the future of each component but cannot infer the relation between them. In other words, type II consciousness can predict or simulate the future but not anticipate the future. As a result, type II consciousness must compensate for lack of anticipation by introducing additional social knowledge, and this could entail either over or underestimate others' future states.

Separation of self's components can play an essential role in anticipation. Sensory attenuation might be based on predicting an unknown future accompanied by the known past. However, that does not mean just matching two states of the known and the unknown. On the one hand, the unknown state is predicted; on the other hand, the known state can add the sense and/or emotion to the predicted state. The ambiguity of unifying and arranging two states can reveal simulation of the state with estimation and emotion and lead to SoA, SoO, and attenuation. Although typical people cannot tickle themselves because their own tickle movements can be predicted, others can tickle them because their movements cannot be predicted. The difference in prediction can entail sensory attenuation (Blakemore et al. 1998). Patients with schizophrenic symptoms (e.g., auditory hallucination) are reported not to show any difference between their own and others' tickling ratings (Blakemore et al. 2000). While the prediction of people with schizophrenia could be damaged, it is important to understand that the ability of prediction and emotional sense such as tickles are interconnected. In our model, type II consciousness also supports such an idea. Because of total weakening of anticipation resulting from isolation of self's components, not only simulation but also accompanied sense and/or emotion becomes dysfunctional, and that can bring about lack of sensory attenuation.

SoA in people with schizophrenia can give us insight into the concept of agency. If the chief in the brain is compared to intentional consciousness, SoA is established by both determinism and free will, combined with loss of locality (i.e., presence of entanglement). Only if preceding readiness potential is entangled with intentional consciousness, intentional consciousness carrying free will claim that the person determines motor representation. While entanglement reveals distributed parallelism, it can also reveal inseparable connection. Therefore, entanglement is neither wholeness reduced to atomic components nor fusion of inseparable elements.

SoA is based on temporal binding (Colonius and Diederich 2004). If action and outcome are coherently perceived, the brain awaits the arrival of the outcome signal to integrate action with outcome. This waiting time is known as the temporal binding window (TBW). If the outcome is predicted and coherently perceived, together with one's voluntary action, TBW becomes narrower. This can lead to discrimination between predicted and 
unpredicted events since only predicted events can fit narrowly modified TBW. Thus, a predicted event is felt as if it occurs simultaneously with voluntary action (i.e., intentional binding) (Haggard et al. 2002a, b; Obhi and Hall 2011). Compared to typical people, TBW for the outcome with more likelihood is not shortened in people with schizophrenia (Voss et al. 2010). This implies that any events can be felt as if those events were outcomes generated by the patient's own action. In other words, SoA is overestimated. This involves patients with schizophrenia having delusions such that daily noise, like a telephone call, could cause independent outcomes (Nathaniel-James and Frith 1996).

Recent studies on cognitive expectation discriminate explicit from implicit expectation. Explicit expectation is expressed as a goal-oriented task, from which the action-outcome can be inferred by the goal. In contrast, in implicit expectation, action-outcome can be connected by prime. While typical people can feel SoA for both explicit and implicit expectation conditions, people with schizophrenia can feel SoA not for implicit but only for the explicit condition (van der Weiden et al. 2013; Renes et al. 2013, 2015). That the patient with schizophrenia feels much more $\mathrm{SoO}$ in contrast to control has also been reported (Thakkar et al. 2011).

It sounds as if these experimental results were inconsistent. With respect to TBW, patients with schizophrenia overestimate SoA; with respect to implicit expectation, they underestimate SoA. This inconsistency can be resolved by type II consciousness in our model. type III consciousness, corresponding to typical people's consciousness, can entangle myself with others in the brain and can entangle prediction with evaluation and emotion. Thus, fitting TBW is accompanied by sense and emotion. Since fitting TBW implies the entanglement of preceding action and following outcome, type III consciousness can bring about emotional judgment to discriminate predicted (e.g., self) and unpredicted events (e.g., others). For predicted events, fit to TBW occurs and underlying emotions can appear. For unpredicted events, fit to TBW does not occur and emotion does not appear.

Compared with type III consciousness, type II consciousness cannot bring about emotion because it can reveal isolation of myself and others in the brain. Fit to TBW could be achieved by ways other than entanglement, that is, by "logical" integration of isolated components in self due to additional knowledge such as social norms and/or social beliefs. Since logical integration can bring about specific sense and emotion, people with type II consciousness can discriminate familiar events (i.e., predicted; self) from unfamiliar events (i.e., unpredicted; others). Although people with type II consciousness never shorten TBW to predicted one, fit to TBW can occur. Thus, the sense or emotion accompanied by self can appear whenever fit to broad TBW occurs and entails overestimation of SoA. In contrast, implicit cognitive expectation never leads to fit to TBW in people with schizophrenia. When fit to TBW does not occur, recruitment of additional knowledge such as social norms and/or social beliefs does not occur. In this sense, any attempts to compensate for entanglement do not occur. Since no emotion like self occurs, misidentification of self and others does not occur, and this implies underestimation of SoA. Finally, our type II consciousness can explain over and underestimation of SoA in people with schizophrenia. More SoO in people with schizophrenia is also consistent with type II consciousness in our model because fusion of self and actual others can embody the persons' own body as an extended body. 


\subsection{Type III Consciousness Carrying Entanglement}

In sections regarding types I and II consciousness, we argue what type III consciousness is compared with types I and II. Here, we add some important aspects of type III consciousness with respect to perception and SoA. Finally, we argue the underlying mechanism suggested by type III consciousness.

\subsubsection{Ignoring Frame Problem}

Through considering type I and type II consciousness, entanglement of type III consciousness can be contrasted to the attribute of the boundary between inside and outside. In type I and type II consciousness, each boundary between inside and outside is either fusion or separation, thus implying either no distinction or explicit separation between inside and outside. Such a boundary cannot implement complex information processing, including meta-processing. As for the inside, the outside cannot be explicitly recognized but can implicitly affect the inside. The frame problem (Dreyfus et al. 2000) and the symbolgrounding problem (Harnad 1990) show that an explicit logical statement and/or symbol must be accompanied by hidden context connected to the real world. While such a context is ignored and hidden, once one seeks explicit meaning or significance of the statement or symbol, it could appear explicitly in an infinite regression manner. Even if you are initially recommended to drink coffee previously unknown to you, you already know that coffee is a beverage. In other words, beverage is a hidden frame accompanying coffee. In this sense, even if coffee is unknown, some aspect of coffee is already known. Specifically, if one never knows any aspect of coffee, then she/he cannot actually comprehend that coffee is unknown. The unknown cannot be perceived until some of its aspects are known.

Such a "frame-problem property" shows us the outside's significance. One can imagine that the outside is logically defined by the negation of the inside. In the sense of cognitive psychology (Lakoff 2008) and the frame problem, the outside implies the context and/or the evaluation at a higher level of the inside. In the sense of frames, "the outside of the outside of "implies the evaluation of states at a lower level. Such consideration entails that the co-existence of the inside and outside implies co-existence of a specific state (the inside) and its evaluation, including emotion (the outside).

The frame-problem property can be extended to general cognition and/or perception. In everyday life, you can see something new that is unknown to you although you already know some of its aspects. If something cannot be recognized at all, you can see neither the unknown nor the known. That is nothing. Ambiguity of known and unknown is a necessary condition for perceiving or recognizing the unknown. In typical people's normal condition, the state of the unknown is implemented by some feeling, sense, and/or emotion of familiarity. In this sense, perceiving the unknown is always accompanied by the known. While the unknown is far from familiar logically, it is accompanied by some familiarity.

Type III consciousness contains the entanglement boundary, which implements an abnormal relation between inside and outside. Entanglement can reveal both parallelism of inside and outside (i.e., separation of inside and outside) and inseparable relation of inside and outside (i.e., fusion). Only type III consciousness can ignore the frame problem. In type I consciousness, inside and outside come together to establish specific states that cannot be specific subjective feeling and/or emotion. Since determinism holds in type I consciousness, the relation among various states can be determined and described. The objective-descriptive world is established from type I consciousness, consistent with the 
consciousness of people with ASD. In type II consciousness, outside is separated from inside. The "unknown" outside of the "known" cannot accompany one another. Thus, type II consciousness cannot allow co-existence of the known and the unknown or the known and the feeling of familiarity. Notwithstanding the separation of inside and outside in type II consciousness, people with schizophrenia communicate with actual others not by feeling and/or emotion but by introducing additional knowledge. Thus, the patient with schizophrenia cannot discriminate himself/herself from others.

\subsubsection{My Agency as Agency of Nobody}

SoA has been considered to be the sense related to prediction ability. The comparator model was originally proposed to discriminate the system's own motion from the motion of the environment surrounding the system (Frith 1992; Blakemore et al. 2000). When matching between the intentional plan to move and motor command is estimated, the extent of matching can reflect the extent of the system's own motion because motion caused by the system itself can be predicted. This idea can be analogous to SoA. As Gallagher (2000) claimed, the comparator model is based on the immune principle of consciousness, i.e., it is assumed that there exists a self that can compare an intentional plan with motor command in advance. We think that is too simple. The self or agent who can control the body cannot exist in advance. Soon after an infant is born, he/she naturally and passively moves arms and legs. An infant does not feel that he/she is the very agent controlling the body because self-consciousness has not yet developed, so the infant has no notion of self. Since an infant can feel that the body is passively moved by somebody, he/she seeks to find somebody in the body (or in the brain). Although somebody makes her/him move, there is nobody in the brain. It results in self as nobody. In other words, finding nobody making her/him move can result in the establishment of self. She/he can be compared to intentional consciousness, and somebody making her/him move can be compared to readiness potential. Since readiness potential is outside intentional consciousness, existence of readiness potential cannot be explicitly found but can be implicitly suggested. That is somebody (readiness potential) making the body move; this is nothing but nobody, except for something equal to intentional consciousness. It is implemented by the entanglement of readiness potential and intentional consciousness and results in sense of self agency. type III consciousness implements the inner and outer boundary equipped with entanglement. It implies that myself (i.e., intentional consciousness), which is explicitly discriminated from others in the brain (i.e., unconsciousness including readiness potential), can be entangled with others in the brain and that self is generated as an entanglement of myself and others in the brain. From the viewpoint of intentional consciousness, voluntary action is already determined by others in the brain. Therefore, myself is passively forced to send "voluntary action." Inferring readiness potential from intentional consciousness implies seeking somebody who makes myself pretend to be active. This could result in failure to find an external agent outside the brain. Thus, "finding nobody" holds true. Finding nobody implies the establishment of self-consciousness or self. Once self is established in the form of entanglement, any preceding information processing is refound as part of self. That is refoundation of self-agency and is SoA. The underlying neural mechanism corresponding to seeking somebody could be global workspace (Dehaene et al. 1998; Dehaene and Naccache 2001; Dehaene and Changeux 2011). In a specific region of the brain, the largest population of neurons is selected and synchronously fired to a given external stimulus. Such selection can correspond to the choice of optimal solution. Thereafter, a specific population 
of neurons as the optimal solution is globally connected to any other neurons employed in various functions, and then some neurons utilize the optimal solution to reveal specific output (i.e., global workspace). That expands a solution with respect to various functions and can correspond to seeking somebody who can achieve a specific intentional move. The failure of seeking somebody and finding self as nobody can correspond to arbitrary usage of the optimal solution. Thus, a pair of choice of optimal solution and expansion to global workspace could imply perpetual establishment of self as nobody.

\subsubsection{Free Will: The Active as the Passive}

Type III consciousness could give insight into the underlying mechanism of postdiction. Since Libet (2004) claimed its significance, comprehending free will as postdictive attribution has been discussed in various fields. Because the declaration "I have free will" is passively enforced by the brain's preceding unconscious domain, free will seems to be an authentic illusion. If so, all researchers have to think about is constructing an authentic illusion for intentional consciousness. Along physical time, voluntary intention (B) follows preceding readiness potential or motor command (A), implying "A then B." However, intentional consciousness himself/herself is confirmed when motor command follows her/his intention, i.e., "B then A." Thus, how to construct an authentic illusion is nothing but how to replace A then B with B then A. Four underlying mechanisms can replace A then B with B then A: catch up, re-entry, different pathways, and memory revision (Shimojo 2014). In the catch up model, information processing for signal "A" or " $B$ " is gradually expanded, and following B catches up preceding A. Thus, the catching-up domain is implemented by mixture of A and B to reveal B then A. In the re-entry model, a copy of lower-level information processing for A and B is transmitted to the higher level; they are re-entered to the lower level, while lower-level information processing proceeds at that very level from A to B. Due to re-entry, re-entered B gets ahead of A, and B then A is achieved. In the different pathways model, while the A signal is transmitted along the slow information processing line, the B signal is transmitted along the fast information processing line. Thus, A then B can be replaced by B then A at the terminal junction of lines (i.e., brain's prefrontal area). In the memory revision model, tentatively established memory on events' order is later revised as another form of the order. These underlying mechanisms show only how to replace A then B with B then A and how to construct a structure of temporal illusion. In contrast, in our model, type III consciousness teaches us loss of locality can intrinsically modify the relationship between free will and determinism. If anything is determined in advance, then one might think that free will does not exist or free will is just an authentic illusion. However, such a thought can hold only if locality holds. If locality holds, multiple information processing occurs, and one information processing can measure another information processing without interference. Only under this condition, myself (intentional consciousness; the one information) can know the motor command that should result from myself was preceded at other places (i.e., there is determinism that can correlate myself and others in the brain). If so, myself can know there is no free will, so that free will, determinism, and locality can constitute trilemma. Since type III consciousness gives up locality, without inference, intentional consciousness cannot know other information processing with any other timing and at any other places. If intentional consciousness accesses other information processing, information itself can be modified and revised. Thus, intentional consciousness or myself cannot see whether her/his own intention is made in advance by others in the brain. Finally, myself cannot determine whether she/he 
has free will. Under loss of locality, intention passively made is paradoxically regarded as active. As mentioned above, finding that nobody controls myself is nothing but a way to establish active myself. That is the only way to justify SoA and/or free will. The final question arises as follows: is locality given up in the trilemma for typical people's consciousness? There is no foundation for loss of locality. However, we examine all possible consciousness options resulting from the trilemma, such as type I (loss of free will), type II (loss of determinism), and type III (loss of locality). Considering all three options, the three types of consciousness could be compared to people with ASD, people with schizophrenia, and typical people. In considering all options, one can see a case without free will. As Dummett mentioned, difference of loss of locality (dancing chief) and loss of determinism (Dummett himself) is just a cultural difference or a difference in interest. Similarly, the difference among typical people and people with ASD and schizophrenia is not an essential human difference but just social interest. As for humans, in some cases, one has free will, and in other cases, no free will. Free will itself is not essential; however, free will is not an illusion. Since trilemma is logical issue, the real consciousness can non-logically reveal mixture of three elements, determinism, free will and locality. While most people reveal one of typical consciousness, type I, II and III, some creative people can have full co-existence of three elements. Such creative people can access all possibilities like type I, feel the universe or space like type III, and positively neglect and fee the outside like type III. That is the essence of creativity, we think.

\section{Conclusion}

The true issue of free will in postdiction is not in inverse causation from future to past but in the context without locality. As Dummett (1978) mentioned, whether forward or backward causation, free will never co-exists with determinism in a normal context in which any other information at different places and in different moments can be accessed without inference. This implies a trilemma among free will, determinism, and locality. The true issue lies in the trilemma. In the trilemma of Dummet's argument, the chief who believes in having free will to begin or stop dancing believes that his dance can make the preceding lion hunt successful (i.e., determinism) because in the universe where the chief lives, there is no locality. This situation resembles the situation in the brain. Intentional consciousness, believing in free will to begin or to stop moving her/his finger, also believes in the ability to determine the motor command that precedes intention and is determined by readiness potential (i.e., determinism), where there is no locality in a neural network universe (i.e., no global super-observer monitors everything in the brain). When the chief and the lion hunt are replaced by intentional consciousness and readiness potential, respectively, an isomorphism is easily seen between the situation of the chief and intentional consciousness. We define the specific rule corresponding to the elements of trilemma, free will, determinism, and locality. Virtual consciousness is defined by self comprising myself and others in the brain accompanied with actual others. Myself and others in the brain can correspond to intentional consciousness and unconsciousness including readiness potential. Actual others correspond to others outside our bodies. Inner and outer boundaries are defined by the boundary between myself and others and by the boundary between self and actual others. Free will and determinism can affect inner and outer boundaries, respectively, where presence of the element creates an explicit boundary and absence of it creates an implicit boundary. Locality can affect both inner and outer boundaries simultaneously, where 
presence of locality can allow the boundary affected by any other elements (free will or determinism) and absence of locality can make boundaries intermediate. These rules bring about three possible types of consciousness: type I, type II, and type III consciousness. Type I consciousness is characterized by inner fusion and outer separation. Therefore, self is separated from actual others, and the body cannot contain any aspects of others. This is consistent with some symptoms of those with ASD, such as weakened theory of mind and weakened embodiment. Type II consciousness is characterized by inner separation and outer fusion. Inner separation implies the separation between myself and others in the brain; this can entail finding others in the self. Type II can reveal an alien hand and/or auditory hallucinations as symptoms in the people with schizophrenia. Outer fusion implies fusion between self and actual others; this can reveal self-other integration and over and underestimation of SoA, typical symptoms of those with schizophrenia. Type III consciousness is characterized by inner and outer intermediate boundaries. It can reveal ambiguity of distinction and integration between myself (self, respectively) and others in the brain (actual others, respectively), that is, entanglement between myself and others. Entanglement of intentional consciousness and unconsciousness can achieve self equipped with free will and reveal SoA. If one considers that SoA can be generated by adequate prediction and/or adequate matching between intentional plan and motor command, one assumes self that can match some states with other states in advance (i.e., immune principle for selfconsciousness). SoA cannot explain until it is explained that self is perpetually generated, degenerated, and sustained. In the scheme of entanglement, after intentional consciousness seeks to find someone who forces her/himself to move, he/she discovers there is nobody. The sign of nobody is itself nothing but self. In other words, self-consciousness is established as nobody in the brain, and generation and degeneration of self cannot appear until entanglement appears.

Acknowledgements We acknowledge JSPS for the financial support of our work, Project Number $17 \mathrm{~K} 18465$.

Open Access This article is distributed under the terms of the Creative Commons Attribution 4.0 International License (http://creativecommons.org/licenses/by/4.0/), which permits unrestricted use, distribution, and reproduction in any medium, provided you give appropriate credit to the original author(s) and the source, provide a link to the Creative Commons license, and indicate if changes were made.

\section{References}

Abu-Akel, A., \& Shamay-Tsoory, S. G. (2013). Characteristics of theory of mind impairments in schizophrenia. In D. L. Roberts \& D. L. Penn (Eds.), Social cognition in schizophrenia: From evidence to treatment (pp. 196-214). New York: Oxford University Press.

Asai, T., Mao, Z., Sugimori, E., \& Tanno, Y. (2011). Rubber hand illusion, empathy, and schizotypal experiences in terms of self-other representations. Consciousness and Cognition, 20(4), 1744-1750.

Baribeau, D. A., \& Anagnostou, E. (2015). Oxytocin and vasopressin: Linking pituitary neuropeptides and their receptors to social neurocircuits. Frontiers in neuroscience, 9, 335.

Baron-Cohen, S. (1991). Precursors to a theory of mind: Understanding attention in others. Natural Theories of Mind: Evolution, Development and Simulation of Everyday Mindreading, 1, 233-251.

Baron-Cohen, S. (1995). Mindblindness: An essay on autism and theory of mind. Cambridge: MIT press.

Baron-Cohen, S., Leslie, A. M., \& Frith, U. (1985). Does the autistic child have a theory of mind? Cognition, 21(1), 37-46.

Blakemore, S. J., Wolpert, D. M., \& Frith, C. D. (1998). Central cancellation of self-produced tickle sensation. Nature Neuroscience, 1(7), 635.

Blakemore, S. J., Wolpert, D., \& Frith, C. (2000). Why can't you tickle yourself? Neuroreport, 11(11), R11-R16. 
Blakemore, S. J., Wolpert, D. M., \& Frith, C. D. (2002). Abnormalities in the awareness of action. Trends in Cognitive Sciences, 6(6), 237-242.

Bleuler, M., \& Bleuler, R. (1986). Dementia praecox oder die gruppe der schizophrenien: Eugen bleuler. The British Journal of Psychiatry, 149(5), 661-664.

Bonini, L., Ferrari, P. F., \& Fogassi, L. (2013). Neurophysiological bases underlying the organization of intentional actions and the understanding of others intention. Consciousness and Cognition, 22(3), 1095-1104.

Botvinick, M. (2004). Probing the neural basis of body ownership. Science, 305(5685), 782-783.

Botvinick, M., \& Cohen, J. (1998). Rubber hands feeltouch that eyes see. Nature, 391(6669), 756.

Brüne, M. (2005). Emotion recognition, theory of mind, and social behavior in schizophrenia. Psychiatry Research, 133(2-3), 135-147.

Carr, L., Iacoboni, M., Dubeau, M. C., Mazziotta, J. C., \& Lenzi, G. L. (2003). Neural mechanisms of empathy in humans: A relay from neural systems for imitation to limbic areas. Proceedings of the National Academy of Sciences, 100(9), 5497-5502.

Carruthers, P. (1996). Simulation and self-knowledge: A defence of the theory-theory. In P. Carruthers \& P. K. Smith (Eds.), Theories of theories of mind. Cambridge: Cambridge University Press.

Cochran, D., Fallon, D., Hill, M., \& Frazier, J. A. (2013). The role of oxytocin in psychiatric disorders: A review of biological and therapeutic research findings. Harvard Review of Psychiatry, 21(5), 219.

Colonius, H., \& Diederich, A. (2004). Multisensory interaction in saccadic reaction time: A time-windowof-integration model. Journal of Cognitive Neuroscience, 16(6), 1000-1009.

Conway, J., \& Kochen, S. (2006). The free will theorem. Foundations of Physics, 36(10), 1441-1473.

Conway, J., \& Kochen, S. (2009). The strong free will theorem. Notices of the AMS, 56(2), 226-232.

Conway, M. A., \& Pleydell-Pearce, C. W. (2000). The construction of autobiographical memories in the self-memory system. Psychological Review, 107(2), 261.

Corbera, S., Wexler, B. E., Ikezawa, S., \& Bell, M. D. (2013). Factor structure of social cognition in schizophrenia: Is empathy preserved? Schizophrenia Research and Treatment, 2013, 409205.

Costantini, M., \& Haggard, P. (2007). The rubber hand illusion: Sensitivity and reference frame for body ownership. Consciousness and Cognition, 16(2), 229-240.

Cunningham, D. W., Billock, V. A., \& Tsou, B. H. (2001). Sensorimotor adaptation to violations of temporal contiguity. Psychological Science, 12(6), 532-535.

Cunningham, S. J., Turk, D. J., Macdonald, L. M., \& Macrae, C. N. (2008). Yours or mine? Ownership and memory. Consciousness and Cognition, 17(1), 312-318.

Cunningham, S. J., Vergunst, F., Macrae, C. N., \& Turk, D. J. (2013). Exploring early self-referential memory effects through ownership. British Journal of Developmental Psychology, 31(3), 289-301.

Dapretto, M., Davies, M. S., Pfeifer, J. H., Scott, A. A., Sigman, M., Bookheimer, S. Y., et al. (2006). Understanding emotions in others: Mirror neuron dysfunction in children with autism spectrum disorders. Nature Neuroscience, 9(1), 28.

David, N., Gawronski, A., Santos, N. S., Huff, W., Lehnhardt, F. G., Newen, A., et al. (2008). Dissociation between key processes of social cognition in autism: Impaired mentalizing but intact sense of agency. Journal of Autism and Developmental Disorders, 38(4), 593-605.

Decety, J., \& Lamm, C. (Eds.). (2011). Empathy versus personal distress: Recent evidence from social neuroscience. In The social neuroscience of empathy (pp. 199-213). Cambridge: The MIT Press.

Dehaene, S., \& Changeux, J. P. (2011). Experimental and theoretical approaches to conscious processing. Neuron, 70(2), 200-227.

Dehaene, S., \& Naccache, L. (2001). Towards a cognitive neuroscience of consciousness: Basic evidence and a workspace framework. Cognition, 79(1-2), 1-37.

Dehaene, S., Kerszberg, M., \& Changeux, J. P. (1998). A neuronal model of a global workspace in effortful cognitive tasks. Proceedings of the National Academy of Sciences, 95(24), 14529-14534.

Dennett, D. C. (1989). The intentional stance. Cambridge: MIT Press.

Dennett, D. C. (2017). Consciousness explained. Brown: Little.

Derntl, B., Finkelmeyer, A., Toygar, T. K., Hülsmann, A., Schneider, F., Falkenberg, D. I., et al. (2009). Generalized deficit in all core components of empathy in schizophrenia. Schizophrenia Research, 108(1-3), 197-206.

Dreyfus, H., Dreyfus, S. E., \& Athanasiou, T. (2000). Mind over machine. New York: Simon and Schuster.

Dummett, M. (1978). Truth and other enigmas. Cambridge: Harvard University Press.

Eagleman, D. M. (2004). The where and when of intention. Science, 303(5661), 1144-1146.

Eagleman, D. M., \& Holcombe, A. O. (2002). Causality and the perception of time. Trends in Cognitive Sciences, 6(8), 323-325. 
Eagleman, D. M., Peter, U. T., Buonomano, D., Janssen, P., Nobre, A. C., \& Holcombe, A. O. (2005). Time and the brain: How subjective time relates to neural time. Journal of Neuroscience, 25(45), 10369-10371.

Ehrsson, H. H., Spence, C., \& Passingham, R. E. (2004). That's my hand! Activity in premotor cortex reflects feeling of ownership of a limb. Science, 305(5685), 875-877.

Enticott, P. G., Bradshaw, J. L., Iansek, R., Tonge, B. J., \& Rinehart, N. J. (2009). Electrophysiological signs of supplementary-motor-area deficits in high-functioning autism but not asperger syndrome: An examination of internally cued movement-related potentials. Developmental Medicine \& Child Neurology, 51(10), 787-791.

Farne, A., Pavani, F., Meneghello, F., \& Ladavas, E. (2000). Left tactile extinction following visual stimulation of a rubber hand. Brain, 123(11), 2350-2360.

Farrer, C., \& Frith, C. D. (2002). Experiencing oneself vs another person as being the cause of an action: The neural correlates of the experience of agency. Neuroimage, 15(3), 596-603.

Ferrari, M., \& Matthews, W. S. (1983). Self-recognition deficits in autism: Syndrome-specific or general developmental delay? Journal of Autism and Developmental Disorders, 13(3), 317-324.

Fett, A. K. J., Viechtbauer, W., Penn, D. L., van Os, J., Krabbendam, L., et al. (2011). The relationship between neurocognition and social cognition with functional outcomes in schizophrenia: A metaanalysis. Neuroscience \& Biobehavioral Reviews, 35(3), 573-588.

Freeman, W. J. (2000). How brains make up their minds. New York: Columbia University Press.

Freeman, W. J., \& Vitiello, G. (2006). Nonlinear brain dynamics as macroscopic manifestation of underlying many-body field dynamics. Physics of Life Reviews, 3(2), 93-118.

Frith, C. (2005). The self in action: Lessons from delusions of control. Consciousness and Cognition, 14(4), 752-770.

Frith, C. D. (1992). The cognitive neuropsychology of schizophrenia. London: Psychology Press.

Frith, C. D., Blakemore, S. J., \& Wolpert, D. M. (2000). Explaining the symptoms of schizophrenia: Abnormalities in the awareness of action. Brain Research Reviews, 31(2-3), 357-363.

Frith, U. (2003). Autism: Explaining the enigma. London: Blackwell Publishing.

Gallagher, S. (2000). Philosophical conceptions of the self: Implications for cognitive science. Trends in Cognitive Sciences, 4(1), 14-21.

Gallagher, S. (2007). The natural philosophy of agency. Philosophy Compass, 2(2), 347-357.

Gallese, V. (2003). The roots of empathy: The shared manifold hypothesis and the neural basis of intersubjectivity. Psychopathology, 36(4), 171-180.

Gallese, V., \& Goldman, A. (1998). Mirror neurons and the simulation theory of mind-reading. Trends in Cognitive Sciences, 2(12), 493-501.

Gallese, V., Fadiga, L., Fogassi, L., \& Rizzolatti, G. (1996). Action recognition in the premotor cortex. Brain, 119(2), 593-609.

Geldard, F. A., \& Sherrick, C. E. (1972). The cutaneous" rabbit": A perceptual illusion. Science, 178(4057), 178-179.

Goldman, A. I. (2009). Mirroring, simulating and mindreading. Mind \& Language, 24(2), $235-252$.

Gopnik, A., \& Wellman, H. M. (1992). Why the child's theory of mind really is a theory. Mind \& Language, 7(1-2), 145-171.

Gould, L. N. (1948). Verbal hallucinations and activity of vocal musculature: An electromygraphic study. American Journal of Psychiatry, 105(5), 367-372.

Gowen, E., \& Hamilton, A. (2013). Motor abilities in autism: A review using a computational context. Journal of Autism and Developmental Disorders, 43(2), 323-344.

Grainger, C., Williams, D. M., \& Lind, S. E. (2014a). Metacognition, metamemory, and mindreading in high-functioning adults with autism spectrum disorder. Journal of Abnormal Psychology, 123(3), 650.

Grainger, C., Williams, D. M., \& Lind, S. E. (2014b). Online action monitoring and memory for selfperformed actions in autism spectrum disorder. Journal of Autism and Developmental Disorders, 44(5), 1193-1206.

Green, M. F., \& Kinsbourne, M. (1990). Subvocal activity and auditory hallucinations: Clues for behavioral treatments? Schizophrenia Bulletin, 16(4), 617-625.

Green, M. F., Bearden, C. E., Cannon, T. D., Fiske, A. P., Hellemann, G. S., Horan, W. P., et al. (2011). Social cognition in schizophrenia, part 1: Performance across phase of illness. Schizophrenia Bulletin, 38(4), 854-864.

Green, M. J., Waldron, J. H., Simpson, I., \& Coltheart, M. (2008). Visual processing of social context during mental state perception in schizophrenia. Journal of Psychiatry \& Neuroscience: JPN, $33(1), 34$. 
Grynszpan, O., Nadel, J., Martin, J. C., Simonin, J., Bailleul, P., Wang, Y., et al. (2012). Self-monitoring of gaze in high functioning autism. Journal of Autism and Developmental Disorders, 42(8), $1642-1650$.

Gunji, Y. P., Minoura, M., Kojima, K., \& Horry, Y. (2017). Free will in Bayesian and inverse Bayesian inference-driven endo-consciousness. Progress in Biophysics and Molecular Biology, 131, $312-324$.

Haggard, P., \& Clark, S. (2003). Intentional action: Conscious experience and neural prediction. Consciousness and Cognition, 12(4), 695-707.

Haggard, P., \& Cole, J. (2007). Intention, attention and the temporal experience of action. Consciousness and Cognition, 16(2), 211-220.

Haggard, P., Aschersleben, G., Gehrke, J., \& Prinz, W. (2002a). Action, binding, and awareness. Oxford: Oxford University Press.

Haggard, P., Clark, S., \& Kalogeras, J. (2002b). Voluntary action and conscious awareness. Nature Neuroscience, 5(4), 382.

Happé, F., \& Frith, U. (1996). The neuropsychology of autism. Brain, 119(4), 1377-1400.

Harnad, S. (1990). The symbol grounding problem. Physica D: Nonlinear Phenomena, 42(1-3), $335-346$.

Hemsley, D. R. (1998). The disruption of the sense of selfin schizophrenia: Potential links with disturbances of information processing. British Journal of Medical Psychology, 71(2), 115-124.

Hill, E., Berthoz, S., \& Frith, U. (2004). Brief report: Cognitive processing of own emotions in individuals with autistic spectrum disorder and in their relatives. Journal of Autism and Developmental Disorders, 34(2), 229-235.

Hobson, R. P. (1995). Autism and the development of mind. London: Psychology Press.

Iacoboni, M. (2009). Imitation, empathy, and mirror neurons. Annual Review of Psychology, 60, 653-670.

Ide, M., \& Wada, M. (2017). Salivary oxytocin concentration associates with the subjective feeling of body ownership during the rubber hand illusion. Frontiers in Human Neuroscience, 11, 166.

Ismail, M. A. F., \& Shimada, S. (2016). Robothand illusion under delayed visual feedback: Relationship between the senses of ownership and agency. PloS One, 11(7), e0159619.

Jardri, R., Delevoye-Turrell, Y., Lucas, B., Pins, D., Bulot, V., Delmaire, C., et al. (2009). Clinical practice of RTMS reveals a functional dissociation between agency and hallucinations in schizophrenia. Neuropsychologia, 47(1), 132-138.

Jardri, R., Pins, D., Lafargue, G., Very, E., Ameller, A., Delmaire, C., et al. (2011). Increased overlap between the brain areas involved in self-other distinction in schizophrenia. PloS One, 6(3), e17500.

Kaiser, M. D., \& Shiffrar, M. (2009). The visual perception of motion by observers with autism spectrum disorders: A review and synthesis. Psychonomic Bulletin \& Review, 16(5), 761-777.

Kalckert, A., \& Ehrsson, H. H. (2014). The moving rubber hand illusion revisited: Comparing movements and visuotactile stimulation to induce illusory ownership. Consciousness and Cognition, 26, 117-132.

Kamitani, Y., \& Shimojo, S. (1999). Manifestation of scotomas created by transcranial magnetic stimulation of human visual cortex. Nature Neuroscience, 2(8), 767.

Kammers, M., de Vignemont, F., Verhagen, L., \& Dijkerman, H. C. (2009). The rubber hand illusion in action. Neuropsychologia, 47(1), 204-211.

Kolers, P. A., \& von Grunau, M. (1975). Visual construction of color is digital. Science, 187(4178), 757-759.

Kolers, P. A., \& von Grünau, M. (1976). Shape and color in apparent motion. Vision Research, 16(4), 329-335.

Kosfeld, M., Heinrichs, M., Zak, P. J., Fischbacher, U., \& Fehr, E. (2005). Oxytocin increases trust in humans. Nature, 435(7042), 673.

Kring, A. M., \& Elis, O. (2013). Emotion deficits in people with schizophrenia. Annual Review of Clinical Psychology, 9, 409-433.

Kring, A. M., Kerr, S. L., \& Earnst, K. S. (1999). Schizophrenic patients show facial reactions to emotional facial expressions. Psychophysiology, 36(2), 186-192.

Kühn, S., Brass, M., \& Haggard, P. (2013). Feeling in control: Neural correlates of experience of agency. Cortex, 49(7), 1935-1942.

Lakoff, G. (2008). Women, fire, and dangerous things. Chicago: University of Chicago press.

Lau, H. C., Rogers, R. D., Haggard, P., \& Passingham, R. E. (2004). Attention to intention. Science, 303(5661), 1208-1210.

Lee, A., Hobson, R. P., \& Chiat, S. (1994). I, you, me, and autism: An experimental study. Journal of Autism and Developmental Disorders, 24(2), 155-176. 
Lefebvre, V. A. (1987). The fundamental structures of human reflexion. Journal of Social and Biological Structures, 10(2), 129-175.

Leslie, A. M. (1991). Theory of mind impairment in autism. In A. Whiten (Ed.), Natural theories of mind: Evolution, development, and simulation of everyday mindreading. Cambridge: Basil Blackwell.

Libet, B. (2004). Mind time: The temporal factor in consciousness. Cambridge: Harvard University Press.

Libet, B., Gleason, C. A., Wright, E. W., \& Pearl, D. K. (1983). Time of conscious intention to act in relation to onset of cerebral activity (readiness-potential). The unconscious initiation of a freely voluntary act. Brain: A Journal of Neurology, 106(Pt 3), 623-42.

Lind, S. E., \& Bowler, D. M. (2009). Recognition memory, self-other source memory, and theory-ofmind in children with autism spectrum disorder. Journal of Autism and Developmental Disorders, 39(9), 1231.

Longo, M. R., Schüür, F., Kammers, M. P., Tsakiris, M., \& Haggard, P. (2008). What is embodiment? A psychometric approach. Cognition, 107(3), 978-998.

Lopatina, O., Inzhutova, A., Salmina, A. B., \& Higashida, H. (2013). The roles of oxytocin and CD38 in social or parental behaviors. Frontiers in Neuroscience, 6, 182.

Louzolo, A., Kalckert, A., \& Petrovic, P. (2015). When passive feels active-delusion-proneness alters selfrecognition in the moving rubber hand illusion. PloS One, 10(6), e0128549.

Markopoulou, F. (2000). The internal description of a causal set: What the universe looks like from the inside. Communications in Mathematical Physics, 211(3), 559-583. https://doi.org/10.1007/s0022 00050826

Martineau, J., Schmitz, C., Assaiante, C., Blanc, R., \& Barthélémy, C. (2004). Impairment of a cortical event-related desynchronisation during a bimanual load-lifting task in children with autistic disorder. Neuroscience Letters, 367(3), 298-303.

McCormick, L. M., Brumm, M. C., Beadle, J. N., Paradiso, S., Yamada, T., \& Andreasen, N. (2012). Mirror neuron function, psychosis, and empathy in schizophrenia. Psychiatry Research: Neuroimaging, 201(3), 233-239.

Mehta, U. M., Thirthalli, J., Aneelraj, D., Jadhav, P., Gangadhar, B. N., \& Keshavan, M. S. (2014). Mirror neuron dysfunction in schizophrenia and its functional implications: A systematic review. Schizophrenia Research, 160(1-3), 9-19.

Mishara, A. L., Lysaker, P. H., \& Schwartz, M. A. (2013). Self-disturbances in schizophrenia: History, phenomenology, and relevant findings from research on metacognition. Schizophrenia Bulletin, 40(1), 5-12.

Montag, C., Heinz, A., Kunz, D., \& Gallinat, J. (2007). Self-reported empathic abilities in schizophrenia. Schizophrenia Research, 92(1-3), 85-89.

Moore, C. (1988). Comment on space-time as a causal set. Physical Review Letters, 60(7), 655.

Moore, J., \& Haggard, P. (2008). Awareness of action: Inference and prediction. Consciousness and Cognition, 17(1), 136-144.

Moore, J. W., \& Obhi, S. S. (2012). Intentional binding and the sense of agency: A review. Consciousness and Cognition, 21(1), 546-561.

Moore, J. W., Ruge, D., Wenke, D., Rothwell, J., \& Haggard, P. (2010). Disrupting the experience of control in the human brain: Pre-supplementary motor area contributes to the sense of agency. Proceedings of the Royal Society B: Biological Sciences, 277, 2503-2509.

Moore, J. W., Teufel, C., Subramaniam, N., Davis, G., \& Fletcher, P. C. (2013). Attribution of intentional causation influences the perception of observed movements: Behavioral evidence and neural correlates. Frontiers in Psychology, 4, 23.

Nathaniel-James, D., \& Frith, C. (1996). Confabulation in schizophrenia: Evidence of a new form? Psychological Medicine, 26(2), 391-399.

Nazarali, N., Glazebrook, C. M., \& Elliott, D. (2009). Movement planning and reprogramming in individuals with autism. Journal of Autism and Developmental Disorders, 39(10), 1401-1411.

Obhi, S. S., \& Hall, P. (2011). Sense of agency and intentional binding in joint action. Experimental Brain Research, 211(3-4), 655.

Ocampo, B., \& Kritikos, A. (2011). Interpreting actions: The goal behind mirror neuron function. Brain Research Reviews, 67(1-2), 260-267.

Paillard, J. (1999). Body schema and body image: A double dissociation in deafferented patients. In G. N. Gantchev, S. Mori, \& J. Massion (Eds.), Motor control, today and tomorrow (pp. 197-214).

Paton, B., Hohwy, J., \& Enticott, P. G. (2012). The rubber hand illusion reveals proprioceptive and sensorimotor differences in autism spectrum disorders. Journal of Autism and Developmental Disorders, 42(9), 1870-1883. 
Perner, J., \& Howes, D. (1992). he thinks he knows: And more developmental evidence against the simulation (role taking) theory. Mind \& Language, 7(1-2), 72-86.

Pinkham, A. E., Penn, D. L., Perkins, D. O., Graham, K. A., \& Siegel, M. (2007). Emotion perception and social skill over the course of psychosis: A comparison of individuals at-risk for psychosis and individuals with early and chronic schizophrenia spectrum illness. Cognitive Neuropsychiatry, 12(3), 198-212.

Premack, D., \& Woodruff, G. (1978). Does the chimpanzee have a theory of mind? Behavioral and Brain Sciences, 1(4), 515-526.

Renes, R. A., Vermeulen, L., Kahn, R. S., Aarts, H., \& van Haren, N. E. (2013). Abnormalities in the establishment of feeling of self-agency in schizophrenia. Schizophrenia Research, 143(1), 50-54.

Renes, R. A., van der Weiden, A., Prikken, M., Kahn, R. S., Aarts, H., \& van Haren, N. E. (2015). Abnormalities in the experience of self-agency in schizophrenia: A replication study. Schizophrenia Research, 164(1-3), 210-213.

Rinehart, N. J., Bellgrove, M. A., Tonge, B. J., Brereton, A. V., Howells-Rankin, D., \& Bradshaw, J. L. (2006). An examination of movement kinematics in young people with high-functioning autism and aspergers disorder: Further evidence for a motor planning deficit. Journal of Autism and Developmental Disorders, 36(6), 757-767.

Riva, G. (2012). Neuroscience and eating disorders: The allocentric lock hypothesis. Medical Hypotheses, $78(2), 254-257$.

Riva, G., \& Gaudio, S. (2012). Allocentric lock in anorexia nervosa: New evidences from neuroimaging studies. Medical Hypotheses, 79(1), 113-117.

Rizzolatti, G., Fadiga, L., Gallese, V., \& Fogassi, L. (1996). Premotor cortex and the recognition of motor actions. Cognitive Brain Research, 3(2), 131-141.

Rogers, K., Dziobek, I., Hassenstab, J., Wolf, O. T., \& Convit, A. (2007). Who cares? Revisiting empathy in asperger syndrome. Journal of Autism and Developmental Disorders, 37(4), 709-715.

Rogers, T. B., Kuiper, N. A., \& Kirker, W. S. (1977). Self-reference and the encoding of personal information. Journal of Personality and Social Psychology, 35(9), 677.

Sakaki, T. (2001). Children with autism, is mind really closed?. Tokyo: PHP Pub. Co.

Sass, L. A. (2014). Self-disturbance and schizophrenia: Structure, specificity, pathogenesis (current issues, new directions). Schizophrenia Research, 152(1), 5-11.

Sass, L. A., \& Parnas, J. (2003). Schizophrenia, consciousness, and the self. Schizophrenia Bulletin, 29(3), 427-444.

Schmitz, C., Martineau, J., Barthélémy, C., \& Assaiante, C. (2003). Motor control and children with autism: Deficit of anticipatory function? Neuroscience Letters, 348(1), 17-20.

Shimojo, S. (2014). Postdiction: Its implications on visual awareness, hindsight, and sense of agency. Frontiers in Psychology, 5, 196.

Smith, M. J., Schroeder, M. P., Abram, S. V., Goldman, M. B., Parrish, T. B., Wang, X., et al. (2014). Alterations in brain activation during cognitive empathy are related to social functioning in schizophrenia. Schizophrenia Bulletin, 41(1), 211-222.

Sorkin, R. (1991). Spacetime and causal sets. In J. D’Olivo, et al. (Eds.), Relating and gravitation: Classical and quantum. Singapore: Word Scientific.

Sperduti, M., Pieron, M., Leboyer, M., \& Zalla, T. (2014). Altered pre-reflective sense of agency in autism spectrum disorders as revealed by reduced intentional binding. Journal of Autism and Developmental Disorders, 44(2), 343-352.

Stetson, C., Cui, X., Montague, P. R., \& Eagleman, D. M. (2006). Motor-sensory recalibration leads to an illusory reversal of action and sensation. Neuron, 51(5), 651-659.

Synofzik, M., Vosgerau, G., \& Newen, A. (2008). Beyond the comparator model: A multifactorial two-step account of agency. Consciousness and Cognition, 17(1), 219-239.

Synofzik, M., Vosgerau, G., \& Voss, M. (2013). The experience of agency: An interplay between prediction and postdiction. Frontiers in Psychology, 4, 127.

Thakkar, K. N., Nichols, H. S., McIntosh, L. G., \& Park, S. (2011). Disturbances in body ownership in schizophrenia: Evidence from the rubber hand illusion and case study of a spontaneous out-of-body experience. PloS One, 6(10), e27089.

Thakkar, K. N., Peterman, J. S., \& Park, S. (2014). Altered brain activation during action imitation and observation in schizophrenia: A translational approach to investigating social dysfunction in schizophrenia. American Journal of Psychiatry, 171(5), 539-548.

Tsakiris, M., \& Haggard, P. (2005). The rubber hand illusion revisited: Visuotactile integration and selfattribution. Journal of Experimental Psychology: Human Perception and Performance, 31(1), 80.

Tsakiris, M., Prabhu, G., \& Haggard, P. (2006). Having a body versus moving your body: How agency structures body-ownership. Consciousness and Cognition, 15(2), 423-432. 
Tse, P. U., Intriligator, J., Rivest, J., \& Cavanagh, P. (2004). Attention and the subjective expansion of time. Perception \& Psychophysics, 66(7), 1171-1189.

Uddin, L. Q. (2011). The self in autism: An emerging view from neuroimaging. Neurocase, 17(3), 201-208.

Utsumi, T. (2015). Psychopathology of autism spectrum disorder, for inherit the stars. Tokyo: PHP Pub. Co.

Van Der Gaag, M. (2006). A neuropsychiatric model of biological and psychological processes in the remission of delusions and auditory hallucinations. Schizophrenia Bulletin, 32(suppl-1), S113-S122.

Varcin, K. J., Bailey, P. E., \& Henry, J. D. (2010). Empathic deficits in schizophrenia: The potential role of rapid facial mimicry. Journal of the International Neuropsychological Society, 16(4), 621-629.

Voss, M., Moore, J., Hauser, M., Gallinat, J., Heinz, A., \& Haggard, P. (2010). Altered awareness of action in schizophrenia: A specific deficit in predicting action consequences. Brain, 133(10), 3104-3112.

Wang, Q. (2001). Culture effects on adults' earliest childhood recollection and self-description: Implications for the relation between memory and the self. Journal of Personality and Social Psychology, 81(2), 220.

van der Weiden, A., Ruys, K. I., \& Aarts, H. (2013). A matter of matching: How goals and primes affect self-agency experiences. Journal of Experimental Psychology: General, 142(3), 954.

van der Weiden, A., Prikken, M., \& van Haren, N. E. (2015). Self-other integration and distinction in schizophrenia: A theoretical analysis and a review of the evidence. Neuroscience \& Biobehavioral Reviews, 57, 220-237.

Wigner, E. P. (1967). Remarks on the mind-body question. In Symmetries and reflections (pp. 171-184). Bloomington: Indiana University Press.

Wigton, R., Jocham Radua, P. A., Averbeck, B., Meyer-Lindenberg, A., McGuire, P., Shergill, S. S., et al. (2015). Neurophysiological effects of acute oxytocin administration: Systematic review and metaanalysis of placebo-controlled imaging studies. Journal of Psychiatry \& Neuroscience: JPN, 40(1), E1.

Williams, D. (2010). Theory of own mind in autism: Evidence of a specific deficit in self-awareness? Autism, 14(5), 474-494.

Williams, D., \& Happé, F. (2010). Representing intentions in self and other: Studies of autism and typical development. Developmental Science, 13(2), 307-319.

Williams, D. M., \& Happé, F. (2009). What did I say? Versus what did I think? Attributing false beliefs to self amongst children with and without autism. Journal of Autism and Developmental Disorders, 39(6), 865-873.

Wolpert, D. M., \& Flanagan, J. R. (2001). Motor prediction. Current Biology, 11(18), R729-R732.

Publisher's Note Springer Nature remains neutral with regard to jurisdictional claims in published maps and institutional affiliations.

Yukio-Pegio Gunji is a professor of Department of Intermedia, Art and Science, School of Fundamental Science and Engineering, Waseda University, Japan. He graduated and got Doctor of Science at Tohoku university, Japan. He firstly studied geology and paleontology, and then shifted to theoretical biology and ethology. He proposed the idea of internal measurement by which an endo-observer can anticiapte the outside of his/ her own perspective. He published 10 books in Japanese and over 200 refereed journal papers.

Kyoko Nakamura is a "Japanese painting" artist. She holds a Ph.D. in Fine Art from Tokyo University of the Arts, Tokyo, Japan. Her research background includes fundamental aspects of the creativity found in life, Human and Nature. She and Yukio published a book in Japanese and English of her own paintings and discourses about creativity, "TANKURI”, Pub. Co., Suiseisha, Japan, in 2018. 